\title{
The Conformational Composition of Cyclopentadienylphosphine Investigated by Microwave Spectroscopy and Quantum Chemical Calculations
}

\section{Supporting Information}

\author{
Harald Møllendal, ${ }^{* \dagger}$ George C. Cole ${ }^{\dagger}$ and Jean-Claude Guillemin ${ }^{\ddagger}$ \\ Department of Chemistry, University of Oslo, Post Office Box 1033 Blindern, NO-0315 Oslo, Norway \\ and Laboratoire de Synthèse et Activation de Biomolécules, Unite Mixte de Recherche 6052 du Centre \\ National de la Recherche Scientifique, Institut de Chimie de Rennes, Ecole Nationale Supérieure de \\ Chimie de Rennes, F-35700 Rennes, France
}

Corresponding author: e-mail harald.mollendal @kjemi.uio.no; tel. 472285 5458; fax +47 22855441

${ }^{\dagger}$ University of Oslo

${ }^{\ddagger}$ Ecole Nationale Supérieure de Chimie de Rennes 
TABLE 1S: Microwave Spectrum of the Ground Vibrational State of Conformer II of Cyclopentadienylphosphine

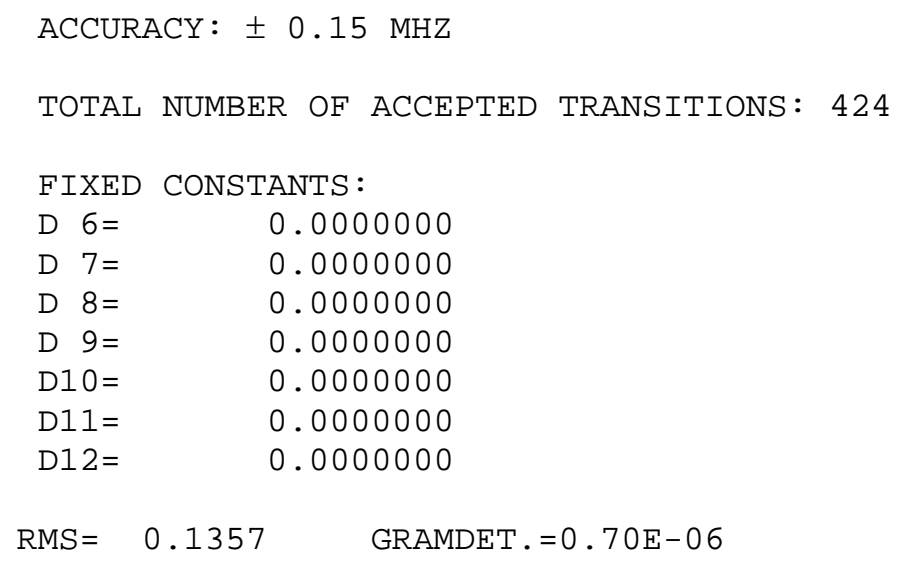

TRANSITION

\begin{tabular}{|c|c|c|c|c|c|}
\hline$g^{\prime \prime}$ & ${ }^{\prime}$ & $1^{\prime}$ & \multicolumn{3}{|c|}{$J^{\prime}{ }_{K-1}{ }_{K+1}^{\prime}$} \\
\hline 6 & 1 & 5 & 7 & 1 & 6 \\
\hline 6 & $\odot$ & 6 & 7 & 1 & 7 \\
\hline 6 & 2 & 4 & 7 & 2 & 5 \\
\hline 6 & 2 & 5 & 7 & 2 & 6 \\
\hline 6 & 3 & 3 & 7 & 3 & 4 \\
\hline 6 & 3 & 4 & 7 & 3 & 5 \\
\hline 6 & 4 & 2 & 7 & 4 & 3 \\
\hline 7 & 3 & 4 & 7 & 4 & \\
\hline 6 & 4 & 3 & 7 & 4 & 4 \\
\hline 7 & 3 & 5 & 7 & 4 & \\
\hline 6 & 5 & 1 & 7 & 5 & 2 \\
\hline 6 & 5 & 2 & 7 & 5 & 3 \\
\hline 6 & 6 & $\odot$ & 7 & 6 & 1 \\
\hline 6 & 6 & 1 & 7 & 6 & 2 \\
\hline 7 & 0 & 7 & 8 & 1 & 8 \\
\hline 7 & 1 & 7 & 8 & 1 & \\
\hline 8 & 3 & 5 & 8 & 4 & 4 \\
\hline 8 & 3 & 6 & 8 & 4 & 5 \\
\hline 7 & 5 & 2 & 8 & 5 & 3 \\
\hline 7 & 5 & 3 & 8 & 5 & 4 \\
\hline 7 & 6 & 1 & 8 & 6 & 2 \\
\hline 7 & 6 & 2 & 8 & 6 & \\
\hline 8 & 6 & 2 & 8 & 7 & 1 \\
\hline 8 & 6 & 3 & 8 & 7 & 2 \\
\hline 8 & 1 & 8 & 9 & 1 & 9 \\
\hline 8 & 3 & 5 & 9 & 3 & 6 \\
\hline 8 & 4 & 4 & 9 & 4 & \\
\hline 8 & 4 & 5 & 9 & 4 & \\
\hline 9 & 3 & 7 & 9 & 4 & 6 \\
\hline 8 & 5 & 3 & 9 & 5 & 4 \\
\hline 8 & 5 & 4 & 9 & 5 & 5 \\
\hline 8 & 6 & 2 & 9 & 6 & 3 \\
\hline 8 & 6 & 3 & 9 & 6 & \\
\hline 9 & 6 & 3 & 9 & 7 & \\
\hline 9 & 6 & 4 & 9 & 7 & \\
\hline
\end{tabular}

$$
\text { OBS. FREQ. O. C. TOTAL }
$$

$\begin{array}{rrr}28978.120 & -0.027 & -0.85 \\ 28449.600 & -0.081 & -0.65 \\ 28892.490 & 0.111 & -0.80 \\ 27919.060 & -0.004 & -0.70 \\ 28299.940 & 0.168 & -0.56 \\ 28205.920 & -0.097 & -0.55 \\ 28183.720 & -0.163 & -0.29 \\ 30567.460 & -0.128 & -0.43 \\ 28181.170 & 0.099 & -0.29 \\ 30720.910 & -0.222 & -0.46 \\ 28152.980 & -0.116 & 0.06 \\ 28152.980 & -0.080 & 0.06 \\ 28137.880 & -0.096 & 0.47 \\ 28137.880 & -0.096 & 0.47 \\ 31716.270 & 0.174 & -1.01 \\ 30368.990 & 0.041 & -1.15 \\ 30373.290 & -0.170 & -0.10 \\ 30702.560 & 0.106 & -0.16 \\ 32191.880 & -0.129 & -0.25 \\ 32191.880 & 0.015 & -0.25 \\ 32169.310 & 0.093 & 0.23 \\ 32169.310 & 0.095 & 0.23 \\ 57167.590 & -0.077 & -7.02 \\ 57167.590 & -0.079 & -7.02 \\ 34088.710 & -0.168 & -1.65 \\ 36616.080 & 0.231 & -1.53 \\ 36310.270 & -0.053 & -1.13 \\ 36291.860 & -0.214 & -1.12 \\ 30702.560 & -0.116 & 0.17 \\ 36237.810 & -0.073 & -0.68 \\ 36237.810 & 0.392 & -0.68 \\ 36205.050 & -\odot .035 & -\odot .14 \\ 36205.050 & -0.029 & -0.14 \\ 57148.870 & 0.072 & -6.39 \\ 57148.870 & 0.064 & -6.39\end{array}$




\begin{tabular}{|c|c|c|c|c|c|c|c|c|}
\hline 9 & 1 & 8 & 10 & 1 & 9 & 40750.470 & -0.163 & -2.38 \\
\hline 9 & 1 & 9 & 10 & 1 & 10 & 37794.480 & 0.131 & -2.26 \\
\hline 9 & 3 & 6 & 10 & 3 & 7 & 40851.310 & $\odot .016$ & -2.25 \\
\hline 9 & 3 & 7 & 10 & 3 & 8 & 40323.820 & 0.111 & -2.09 \\
\hline 9 & 4 & 5 & 10 & 4 & 6 & 40398.730 & -0.133 & -1.76 \\
\hline 10 & 3 & 7 & 10 & 4 & 6 & 29615.200 & $-\odot .303$ & 0.79 \\
\hline 10 & 3 & 8 & 10 & 4 & 7 & 30739.040 & 0.192 & 0.51 \\
\hline 10 & 6 & 4 & 10 & 7 & 3 & 57122.820 & 0.112 & -5.69 \\
\hline 10 & 6 & 5 & 10 & 7 & 4 & 57122.820 & $\odot .082$ & -5.69 \\
\hline 10 & $\odot$ & 10 & 11 & $\odot$ & 11 & 41639.930 & 0.008 & -3.00 \\
\hline 10 & 11 & 10 & 11 & $\odot$ & 11 & 41186.800 & 0.176 & -3.14 \\
\hline 10 & 2 & 9 & 11 & 1 & 10 & 39284.980 & -0.216 & -4.02 \\
\hline 10 & 11 & 10 & 11 & 1 & 11 & 41487.980 & 0.034 & -3.02 \\
\hline 10 & 4 & 6 & 11 & 4 & 7 & 44510.810 & -0.078 & -2.56 \\
\hline 11 & 3 & 8 & 11 & 4 & 7 & 28986.080 & -0.286 & 1.38 \\
\hline 11 & 3 & 9 & 11 & 4 & 8 & 30831.580 & -0.186 & 0.87 \\
\hline 10 & 5 & 5 & 11 & 5 & 6 & 44354.910 & -0.076 & -1.98 \\
\hline 11 & 4 & 7 & 11 & 5 & 6 & 39151.480 & 0.014 & 0.11 \\
\hline 10 & 5 & 6 & 11 & 5 & 7 & 44351.840 & $\odot .069$ & -1.98 \\
\hline 10 & 6 & 4 & 11 & 6 & 5 & 44293.140 & 0.015 & -1.32 \\
\hline 10 & 6 & 5 & 11 & 6 & 6 & 44293.140 & $\odot .090$ & -1.32 \\
\hline 10 & 7 & 3 & 11 & 7 & 4 & 44258.010 & -0.134 & -0.54 \\
\hline 11 & 6 & 5 & 11 & 7 & 4 & 57087.960 & 0.233 & -4.91 \\
\hline 10 & 7 & 4 & 11 & 7 & 5 & 44258.010 & -0.133 & -0.54 \\
\hline 11 & 6 & 6 & 11 & 7 & 5 & 57087.960 & 0.129 & -4.91 \\
\hline 10 & 9 & 1 & 11 & 9 & 2 & 44221.620 & $-\odot .089$ & 1.36 \\
\hline 10 & 9 & 2 & 11 & 9 & 3 & 44221.620 & $-\odot .089$ & 1.36 \\
\hline 10 & 10 & $\odot$ & 11 & 10 & 1 & 44211.640 & -0.172 & 2.48 \\
\hline 10 & 10 & 1 & 11 & 10 & 2 & 44211.640 & -0.172 & 2.48 \\
\hline 11 & 1 & 11 & 12 & $\odot$ & 12 & 44974.980 & -0.015 & -4.03 \\
\hline 11 & 21 & 10 & 12 & 1 & 11 & 44055.450 & -0.126 & -5.00 \\
\hline 12 & 3 & 9 & 12 & 4 & 8 & 28167.740 & -0.150 & 2.07 \\
\hline 12 & 31 & 10 & 12 & 4 & 9 & 31004.750 & $-\odot .064$ & 1.21 \\
\hline 12 & 4 & 9 & 12 & 5 & 8 & 39199.700 & -0.102 & 0.64 \\
\hline 12 & 6 & 6 & 12 & 7 & 5 & 57042.050 & $\odot .079$ & -4.06 \\
\hline 12 & 6 & 7 & 12 & 7 & 6 & 57042.050 & -0.231 & -4.06 \\
\hline 12 & 1 & 11 & 13 & 2 & 12 & 54248.480 & 0.041 & -3.85 \\
\hline 12 & 21 & 11 & 13 & 2 & 12 & 51045.160 & -0.140 & -4.95 \\
\hline 13 & 11 & 13 & 13 & 2 & 12 & 29449.520 & -0.144 & 0.44 \\
\hline 13 & 31 & 10 & 13 & 4 & 9 & 27175.990 & ๑. 089 & 2.83 \\
\hline 12 & 4 & 9 & 13 & 4 & 10 & 52594.000 & 0.109 & -4.62 \\
\hline 13 & 31 & 11 & 13 & 4 & 10 & 31282.750 & 0.266 & 1.53 \\
\hline 12 & 5 & 7 & 13 & 5 & 8 & 52515.920 & $-\odot .067$ & -4.00 \\
\hline 12 & 5 & 8 & 13 & 5 & 9 & 52500.650 & $-\odot .096$ & -3.99 \\
\hline 13 & 41 & 10 & 13 & 5 & 9 & 39106.480 & -0.177 & 1.27 \\
\hline 12 & 7 & 5 & 13 & 7 & 6 & 52348.660 & 0.126 & -2.26 \\
\hline 12 & 7 & 6 & 13 & 7 & 7 & 52348.660 & 0.137 & -2.26 \\
\hline 12 & 8 & 4 & 13 & 8 & 5 & 52311.840 & 0.054 & -1.20 \\
\hline 12 & 8 & 5 & 13 & 8 & 6 & 52311.840 & 0.054 & -1.20 \\
\hline 12 & 9 & 3 & 13 & 9 & 4 & 52287.610 & 0.263 & -0.01 \\
\hline 12 & 9 & 4 & 13 & 9 & 5 & 52287.610 & $\odot .263$ & -0.01 \\
\hline 12 & 10 & 2 & 13 & 10 & 3 & 52270.550 & $\odot .080$ & 1.32 \\
\hline 12 & 10 & 3 & 13 & 10 & 4 & 52270.550 & $\odot . \odot 8 \odot$ & 1.32 \\
\hline 12 & 11 & 1 & 13 & 11 & 2 & 52258.610 & $\odot .079$ & 2.79 \\
\hline 12 & 11 & 2 & 13 & 11 & 3 & 52258.610 & 0.079 & 2.79 \\
\hline 12 & 12 & $\odot$ & 13 & 12 & 1 & 52249.960 & -0.016 & 4.40 \\
\hline 12 & 12 & 1 & 13 & 12 & 2 & 52249.960 & -0.016 & 4.40 \\
\hline
\end{tabular}




\begin{tabular}{|c|c|c|c|c|c|c|c|c|}
\hline 13 & 1 & 13 & 14 & $\odot$ & 14 & 52439.700 & -0.088 & -6.30 \\
\hline 13 & 2 & 12 & 14 & 1 & 13 & 53067.290 & -0.116 & -7.24 \\
\hline 13 & $\odot$ & 13 & 14 & 1 & 14 & 52648.690 & 0.032 & -6.15 \\
\hline 13 & 3 & 10 & 14 & 3 & 11 & 58175.140 & 0.156 & -7.03 \\
\hline 14 & 2 & 13 & 14 & 3 & 12 & 29059.380 & 0.017 & 0.74 \\
\hline 13 & 4 & 9 & 14 & 4 & 10 & $57059.80 \odot$ & -0.112 & -6.25 \\
\hline 14 & 3 & 11 & 14 & 4 & 10 & 26060.960 & 0.131 & 3.61 \\
\hline 13 & 4 & 10 & 14 & 4 & 11 & 56671.480 & -0.051 & -5.95 \\
\hline 13 & 5 & 8 & 14 & 5 & 9 & 56618.520 & -0.003 & -5.32 \\
\hline 14 & 4 & 10 & 14 & 5 & 9 & 38168.360 & -0.285 & 2.47 \\
\hline 13 & 5 & 9 & 14 & 5 & 10 & 56588.660 & 0.089 & -5.30 \\
\hline 14 & 4 & 11 & 14 & 5 & 10 & 39023.680 & -0.018 & 1.92 \\
\hline 13 & 6 & 7 & 14 & 6 & 8 & 56475.790 & -0.072 & -4.43 \\
\hline 13 & 6 & 8 & 14 & 6 & 9 & 56474.650 & 0.046 & -4.43 \\
\hline 13 & 7 & 6 & 14 & 7 & 7 & 56401.800 & 0.029 & -3.42 \\
\hline 14 & 6 & 8 & 14 & 7 & 7 & 56909.220 & 0.021 & -2.13 \\
\hline 13 & 7 & 7 & 14 & 7 & 8 & 56401.800 & 0.062 & -3.42 \\
\hline 14 & 6 & 9 & 14 & 7 & 8 & 56911.270 & 0.014 & -2.13 \\
\hline 13 & 8 & 5 & 14 & 8 & 6 & 56355.420 & -0.156 & -2.28 \\
\hline 13 & 8 & 6 & 14 & 8 & 7 & 56355.420 & -0.155 & -2.28 \\
\hline 13 & 9 & 4 & 14 & 9 & 5 & 56324.860 & 0.052 & -0.99 \\
\hline 13 & 9 & 5 & 14 & 9 & 6 & 56324.860 & 0.052 & -0.99 \\
\hline 13 & 10 & 3 & 14 & 10 & 4 & 56303.570 & 0.084 & 0.45 \\
\hline 13 & 10 & 4 & 14 & 10 & 5 & 56303.570 & 0.084 & 0.45 \\
\hline 13 & 11 & 2 & 14 & 11 & 3 & 56288.170 & -0.146 & 2.03 \\
\hline 13 & 11 & 3 & 14 & 11 & 4 & 56288.170 & -0.146 & 2.03 \\
\hline 13 & 12 & 1 & 14 & 12 & 2 & $56277.40 \odot$ & 0.048 & 3.76 \\
\hline 13 & 12 & 2 & 14 & 12 & 3 & 56277.400 & 0.048 & 3.76 \\
\hline 13 & 13 & 0 & 14 & 13 & 1 & 56269.640 & 0.261 & 5.64 \\
\hline 13 & 13 & 1 & 14 & 13 & 2 & 56269.640 & 0.261 & 5.64 \\
\hline 14 & $\odot$ & 14 & 15 & 0 & 15 & 56219.340 & 0.097 & -7.66 \\
\hline 14 & 1 & 14 & 15 & 0 & 15 & 56137.720 & -0.066 & -7.73 \\
\hline 14 & 1 & 13 & 15 & 1 & 14 & 59051.610 & 0.129 & -7.51 \\
\hline 14 & $\odot$ & 14 & 15 & 1 & 15 & 56270.950 & 0.095 & -7.61 \\
\hline 14 & 1 & 14 & 15 & 1 & 15 & 56189.250 & -0.148 & -7.68 \\
\hline 14 & 1 & 13 & 15 & 2 & 14 & 60286.610 & -0.155 & -6.55 \\
\hline 15 & 1 & 15 & 15 & 2 & 14 & 34093.340 & 0.148 & 0.56 \\
\hline 14 & 4 & 10 & 15 & 4 & 11 & 61339.500 & 0.284 & -7.99 \\
\hline 15 & 3 & 12 & 15 & 4 & 11 & 24905.610 & -0.083 & 4.31 \\
\hline 14 & 4 & 11 & 15 & 4 & 12 & 60741.420 & -0.285 & -7.50 \\
\hline 14 & 5 & 9 & 15 & 5 & 10 & 60740.620 & 0.363 & -6.89 \\
\hline 15 & 4 & 11 & 15 & 5 & 10 & 37569.480 & -0.207 & 3.57 \\
\hline 14 & 5 & 10 & 15 & 5 & 11 & 60684.290 & -0.306 & -6.83 \\
\hline 15 & 4 & 12 & 15 & 5 & 11 & 38966.480 & -0.109 & 2.59 \\
\hline 14 & 6 & 8 & 15 & 6 & 9 & 60553.410 & -0.025 & -5.90 \\
\hline 14 & 6 & 9 & 15 & 6 & 10 & 60550.500 & -0.171 & -5.90 \\
\hline 14 & 7 & 7 & 15 & 7 & 8 & 60461.020 & 0.020 & -4.81 \\
\hline 15 & 6 & 9 & 15 & 7 & 8 & 56816.590 & -0.174 & -1.03 \\
\hline 14 & 7 & 8 & 15 & 7 & 9 & 60461.020 & 0.106 & -4.81 \\
\hline 14 & 8 & 6 & 15 & 8 & 7 & 60403.640 & -0.168 & -3.57 \\
\hline 14 & 8 & 7 & 15 & 8 & 8 & 60403.640 & -0.166 & -3.57 \\
\hline 14 & 9 & 5 & 15 & 9 & 6 & 60365.720 & 0.021 & -2.19 \\
\hline 14 & 9 & 6 & 15 & 9 & 7 & 60365.720 & 0.021 & -2.19 \\
\hline 14 & 10 & 4 & 15 & 10 & 5 & 60339.150 & -0.073 & -0.65 \\
\hline 14 & 10 & 5 & 15 & 10 & 6 & 60339.150 & -0.073 & -0.65 \\
\hline 14 & 11 & 3 & 15 & 11 & 4 & 60320.270 & -0.035 & 1.05 \\
\hline 14 & 11 & 4 & 15 & 11 & 5 & 60320.270 & -0.035 & 1.05 \\
\hline
\end{tabular}




\begin{tabular}{|c|c|c|c|c|c|c|c|c|}
\hline 14 & 12 & 2 & 15 & 12 & 3 & 60306.670 & 0.129 & 2.91 \\
\hline 14 & 12 & 3 & 15 & 12 & 4 & $60306.67 \odot$ & 0.129 & 2.91 \\
\hline 14 & 13 & 1 & 15 & 13 & 2 & $60296.49 \odot$ & $\odot .055$ & 4.92 \\
\hline 14 & 13 & 2 & 15 & 13 & 3 & $6 \odot 296.49 \odot$ & 0.055 & 4.92 \\
\hline 14 & 14 & $\odot$ & 15 & 14 & 1 & 60289.120 & 0.108 & 7.10 \\
\hline 14 & 14 & 1 & 15 & 14 & 2 & 60289.120 & 0.108 & 7.10 \\
\hline 15 & 0 & 15 & 16 & 0 & 16 & 59873.850 & -0.204 & -9.31 \\
\hline 15 & 1 & 15 & 16 & $\odot$ & 16 & 59822.570 & 0.128 & -9.36 \\
\hline 15 & 1 & 15 & 16 & 1 & 16 & 59854.920 & 0.025 & -9.32 \\
\hline 16 & 1 & 15 & 16 & 2 & 14 & $26334.07 \odot$ & $-\odot .201$ & -2.89 \\
\hline 16 & 1 & 16 & 16 & 2 & 15 & 36506.060 & $-\odot .022$ & 0.66 \\
\hline 16 & 2 & 15 & 16 & 3 & 14 & 32503.350 & 0.275 & 0.52 \\
\hline 15 & 4 & 12 & 16 & 4 & 13 & 64799.370 & 0.038 & -9.25 \\
\hline 16 & 4 & 12 & 16 & 5 & 11 & 36782.720 & $\odot .09 \odot$ & 4.88 \\
\hline 15 & 5 & 11 & 16 & 5 & 12 & 64787.420 & $-\odot .088$ & -8.61 \\
\hline 15 & 6 & 9 & 16 & 6 & 10 & 64640.940 & -0.133 & -7.61 \\
\hline 15 & 6 & 10 & 16 & 6 & 11 & 64635.180 & -0.165 & -7.61 \\
\hline 15 & 7 & 8 & 16 & 7 & 9 & $64526.69 \odot$ & $-\odot .039$ & -6.43 \\
\hline 16 & 6 & 10 & 16 & 7 & 9 & 56702.470 & ๑. 050 & $\odot .15$ \\
\hline 15 & 7 & 9 & 16 & 7 & 10 & $64526.69 \odot$ & 0.168 & -6.43 \\
\hline 16 & 6 & 11 & 16 & 7 & 10 & 56712.720 & 0.044 & 0.13 \\
\hline 15 & 8 & 7 & 16 & 8 & 8 & 64456.710 & -0.115 & -5.11 \\
\hline 15 & 8 & 8 & 16 & 8 & 9 & 64456.710 & -0.110 & -5.11 \\
\hline 15 & 9 & 6 & 16 & 9 & 7 & 64410.110 & -0.166 & -3.63 \\
\hline 15 & 9 & 7 & 16 & 9 & 8 & 64410.110 & $-\odot .166$ & -3.63 \\
\hline 15 & 10 & 5 & 16 & 10 & 6 & 64377.850 & -0.030 & -1.98 \\
\hline 15 & 10 & 6 & 16 & 10 & 7 & 64377.850 & $-\odot .030$ & -1.98 \\
\hline 15 & 11 & 4 & 16 & 11 & 5 & 64354.700 & 0.044 & $-\odot .16$ \\
\hline 15 & 11 & 5 & 16 & 11 & 6 & 64354.700 & 0.044 & -0.16 \\
\hline 15 & 12 & 3 & 16 & 12 & 4 & 64337.690 & 0.018 & 1.82 \\
\hline 15 & 12 & 4 & 16 & 12 & 5 & 64337.690 & 0.018 & 1.82 \\
\hline 15 & 13 & 2 & 16 & 13 & 3 & 64325.220 & 0.112 & 3.97 \\
\hline 15 & 13 & 3 & 16 & 13 & 4 & 64325.220 & 0.112 & 3.97 \\
\hline 15 & 14 & 1 & 16 & 14 & 2 & 64315.720 & $-\odot .059$ & 6.29 \\
\hline 15 & 14 & 2 & 16 & 14 & 3 & 64315.720 & $-\odot .059$ & 6.29 \\
\hline 15 & 15 & 0 & 16 & 15 & 1 & $64309.07 \odot$ & 0.182 & 8.79 \\
\hline 15 & 15 & 1 & 16 & 15 & 2 & 64309.070 & 0.182 & 8.79 \\
\hline 17 & 1 & 17 & 17 & 2 & 16 & 38960.420 & 0.083 & 0.80 \\
\hline 17 & 2 & 16 & 17 & 3 & 15 & 34460.880 & $-\odot .082$ & 0.36 \\
\hline 16 & 4 & 13 & 17 & 4 & 14 & 68839.190 & -0.111 & -11.23 \\
\hline 17 & 3 & 15 & 17 & 4 & 14 & 33879.910 & -0.033 & 2.08 \\
\hline 16 & 5 & 11 & 17 & 5 & 12 & 69061.550 & -0.051 & -10.87 \\
\hline 17 & 4 & 13 & 17 & 5 & 12 & 35789.160 & $-\odot .287$ & 6.40 \\
\hline 16 & 5 & 12 & 17 & 5 & 13 & 68895.110 & -0.112 & -10.63 \\
\hline 17 & 4 & 14 & 17 & 5 & 13 & 39010.730 & 0.043 & 3.84 \\
\hline 16 & 6 & 10 & 17 & 6 & 11 & 68740.240 & 0.019 & -9.60 \\
\hline 16 & 6 & 11 & 17 & 6 & 12 & 68729.060 & 0.108 & -9.58 \\
\hline 16 & 7 & 9 & 17 & 7 & 10 & 68599.370 & -0.141 & -8.32 \\
\hline 16 & 7 & 10 & 17 & 7 & 11 & 68599.370 & 0.331 & -8.32 \\
\hline 17 & 6 & 12 & 17 & 7 & 11 & 56582.890 & 0.127 & 1.39 \\
\hline 16 & 8 & 8 & 17 & 8 & 9 & $68515.00 \odot$ & 0.018 & -6.90 \\
\hline 16 & 8 & 9 & 17 & 8 & 10 & $68515.00 \odot$ & $\odot .032$ & -6.90 \\
\hline 16 & 9 & 7 & 17 & 9 & 8 & 68458.840 & 0.040 & -5.31 \\
\hline 16 & 9 & 8 & 17 & 9 & 9 & 68458.840 & 0.040 & -5.31 \\
\hline 16 & 10 & 6 & 17 & 10 & 7 & 68419.530 & -0.130 & -3.56 \\
\hline 16 & 10 & 7 & 17 & 10 & 8 & 68419.530 & -0.130 & -3.56 \\
\hline 16 & 11 & 5 & 17 & 11 & 6 & 68391.620 & $\odot .089$ & -1.63 \\
\hline
\end{tabular}




\begin{tabular}{|c|c|c|c|c|c|c|c|c|}
\hline 16 & 11 & 6 & 17 & 11 & 7 & 68391.620 & $\odot .089$ & -1.63 \\
\hline 16 & 12 & 4 & 17 & 12 & 5 & 68370.950 & 0.072 & 0.48 \\
\hline 16 & 12 & 5 & 17 & 12 & 6 & 68370.950 & 0.072 & 0.48 \\
\hline 16 & 13 & 3 & 17 & 13 & 4 & 68355.410 & $-\odot .098$ & 2.77 \\
\hline 16 & 13 & 4 & 17 & 13 & 5 & 68355.410 & $-\odot .098$ & 2.77 \\
\hline 16 & 14 & 2 & 17 & 14 & 3 & 68344.040 & 0.042 & 5.24 \\
\hline 16 & 14 & 3 & 17 & 14 & 4 & 68344.040 & 0.042 & 5.24 \\
\hline 16 & 15 & 1 & 17 & 15 & 2 & $68335.37 \odot$ & $-\odot .020$ & 7.89 \\
\hline 16 & 15 & 2 & 17 & 15 & 3 & 68335.370 & -0.020 & 7.89 \\
\hline 16 & 16 & 0 & 17 & 16 & 1 & 68329.050 & $\odot .029$ & 10.72 \\
\hline 16 & 16 & 1 & 17 & 16 & 2 & 68329.050 & 0.029 & 10.72 \\
\hline 18 & 1 & 17 & 18 & 2 & 16 & 32663.150 & 0.147 & -3.56 \\
\hline 18 & 2 & 17 & 18 & 3 & 16 & 36552.610 & 0.032 & 0.17 \\
\hline 18 & 3 & 16 & 18 & 4 & 15 & 34980.170 & 0.128 & 1.93 \\
\hline 18 & 4 & 14 & 18 & 5 & 13 & 34595.730 & -0.075 & 8.06 \\
\hline 18 & 4 & 15 & 18 & 5 & 14 & 39158.780 & 0.085 & 4.35 \\
\hline 17 & 7 & 10 & 18 & 7 & 11 & 72679.800 & -0.164 & -10.48 \\
\hline 18 & 6 & 12 & 18 & 7 & 11 & 56389.170 & 0.268 & 2.81 \\
\hline 17 & 7 & 11 & 18 & 7 & 12 & 72678.990 & 0.044 & -10.48 \\
\hline 18 & 6 & 13 & 18 & 7 & 12 & 56429.970 & -0.144 & 2.74 \\
\hline 17 & 8 & 9 & 18 & 8 & 10 & 72578.520 & -0.126 & -8.96 \\
\hline 17 & 8 & 10 & 18 & 8 & 11 & 72578.520 & -0.093 & -8.96 \\
\hline 17 & 9 & 8 & 18 & 9 & 9 & 72511.590 & 0.053 & -7.27 \\
\hline 17 & 9 & 9 & 18 & 9 & 10 & 72511.590 & 0.053 & -7.27 \\
\hline 17 & 10 & 7 & 18 & 10 & 8 & 72464.690 & $-\odot .077$ & -5.41 \\
\hline 17 & 10 & 8 & 18 & 10 & 9 & 72464.690 & -0.077 & -5.41 \\
\hline 17 & 11 & 6 & 18 & 11 & 7 & $72431.04 \odot$ & -0.052 & -3.36 \\
\hline 17 & 11 & 7 & 18 & 11 & 8 & 72431.040 & -0.052 & -3.36 \\
\hline 17 & 12 & 5 & 18 & 12 & 6 & 72406.370 & 0.081 & -1.12 \\
\hline 17 & 12 & 6 & 18 & 12 & 7 & 72406.370 & 0.081 & -1.12 \\
\hline 17 & 13 & 4 & 18 & 13 & 5 & $72387.67 \odot$ & $-\odot .073$ & 1.31 \\
\hline 17 & 13 & 5 & 18 & 13 & 6 & 72387.670 & -0.073 & 1.31 \\
\hline 17 & 14 & 3 & 18 & 14 & 4 & 72373.730 & -0.030 & 3.92 \\
\hline 17 & 14 & 4 & 18 & 14 & 5 & 72373.730 & -0.030 & 3.92 \\
\hline 17 & 15 & 2 & 18 & 15 & 3 & 72363.070 & -0.131 & 6.73 \\
\hline 17 & 15 & 3 & 18 & 15 & 4 & 72363.070 & -0.131 & 6.73 \\
\hline 17 & 16 & 1 & 18 & 16 & 2 & 72355.320 & 0.043 & 9.73 \\
\hline 17 & 16 & 2 & 18 & 16 & 3 & 72355.320 & 0.043 & 9.73 \\
\hline 17 & 17 & 0 & 18 & 17 & 1 & 72349.520 & 0.095 & 12.92 \\
\hline 17 & 17 & 1 & 18 & 17 & 2 & 72349.520 & 0.095 & 12.92 \\
\hline 19 & 2 & 17 & 19 & 3 & 16 & 24676.450 & $-\odot .001$ & -4.76 \\
\hline 19 & 3 & 17 & 19 & 4 & 16 & 36274.630 & 0.129 & 1.66 \\
\hline 19 & 4 & 15 & 19 & 5 & 14 & $3324 \odot .27 \odot$ & 0.206 & 9.74 \\
\hline 19 & 4 & 16 & 19 & 5 & 15 & 39423.370 & -0.237 & 4.73 \\
\hline 19 & 6 & 13 & 19 & 7 & 12 & 56176.620 & 0.156 & 4.33 \\
\hline 18 & 7 & 12 & 19 & 7 & 13 & 76766.630 & $-\odot .080$ & -12.93 \\
\hline 19 & 6 & 14 & 19 & 7 & 13 & 56253.650 & -0.112 & 4.18 \\
\hline 18 & 8 & 10 & 19 & 8 & 11 & 76648.100 & -0.106 & -11.31 \\
\hline 18 & 8 & 11 & 19 & 8 & 12 & 76648.100 & -0.028 & -11.31 \\
\hline 18 & 9 & 9 & 19 & 9 & 10 & 76568.780 & 0.019 & -9.52 \\
\hline 18 & 9 & 10 & 19 & 9 & 11 & 76568.780 & 0.021 & -9.52 \\
\hline 18 & 10 & 8 & 19 & 10 & 9 & 76513.550 & 0.140 & -7.54 \\
\hline 18 & 10 & 9 & 19 & 10 & 10 & 76513.550 & 0.140 & -7.54 \\
\hline 18 & 11 & 7 & 19 & 11 & 8 & 76473.370 & -0.134 & -5.37 \\
\hline 18 & 11 & 8 & 19 & 11 & 9 & 76473.370 & -0.134 & -5.37 \\
\hline 18 & 12 & 6 & 19 & 12 & 7 & 76444.210 & 0.171 & -3.00 \\
\hline 18 & 12 & 7 & 19 & 12 & 8 & 76444.210 & 0.171 & -3.00 \\
\hline
\end{tabular}




\begin{tabular}{|c|c|c|c|c|c|c|c|c|}
\hline 18 & 13 & 5 & 19 & 13 & 6 & 76421.910 & -0.013 & -0.44 \\
\hline 18 & 13 & 6 & 19 & 13 & 7 & 76421.910 & -0.013 & -0.44 \\
\hline 18 & 14 & 4 & 19 & 14 & 5 & 76405.020 & -0.138 & 2.33 \\
\hline 18 & 14 & 5 & 19 & 14 & 6 & 76405.020 & $-\odot .138$ & 2.33 \\
\hline 18 & 15 & 3 & 19 & 15 & 4 & 76392.420 & 0.021 & 5.29 \\
\hline 18 & 15 & 4 & 19 & 15 & 5 & 76392.420 & $\odot .021$ & 5.29 \\
\hline 18 & 16 & 2 & 19 & 16 & 3 & 76382.800 & ๑. 083 & 8.46 \\
\hline 18 & 16 & 3 & 19 & 16 & 4 & 76382.800 & 0.083 & 8.46 \\
\hline 18 & 17 & 1 & 19 & 17 & 2 & 76375.520 & 0.070 & 11.83 \\
\hline 18 & 17 & 2 & 19 & 17 & 3 & 76375.520 & 0.070 & 11.83 \\
\hline 20 & 1 & 19 & 20 & 2 & 18 & 38869.640 & $\odot .001$ & -3.49 \\
\hline 20 & 2 & 18 & 20 & 3 & 17 & 27546.560 & -0.221 & -6.51 \\
\hline 20 & 2 & 19 & 20 & 3 & 18 & 41058.420 & $\odot .242$ & -0.20 \\
\hline 20 & 4 & 16 & 20 & 5 & 15 & 31795.980 & $\odot .009$ & 11.24 \\
\hline 20 & 4 & 17 & 20 & 5 & 16 & 39829.290 & 0.097 & 4.95 \\
\hline 20 & 6 & 14 & 20 & 7 & 13 & 55914.220 & -0.161 & 6.00 \\
\hline 20 & 6 & 15 & 20 & 7 & 14 & 56053.960 & 0.129 & 5.72 \\
\hline 19 & 9 & 10 & 20 & 9 & 11 & 80630.770 & 0.016 & -12.07 \\
\hline 19 & 9 & 11 & 20 & 9 & 12 & 80630.770 & 0.021 & -12.07 \\
\hline 19 & 10 & 9 & 20 & 10 & 10 & 80565.770 & $-\odot .029$ & -9.98 \\
\hline 19 & 10 & 10 & 20 & 10 & 11 & $80565.77 \odot$ & $-\odot .029$ & -9.98 \\
\hline 19 & 11 & 8 & 20 & 11 & 9 & 80518.930 & $-\odot .003$ & -7.68 \\
\hline 19 & 11 & 9 & 20 & 11 & 10 & 80518.930 & -0.003 & -7.68 \\
\hline 19 & 15 & 4 & 20 & 15 & 5 & 80423.120 & $\odot .060$ & 3.56 \\
\hline 19 & 15 & 5 & 20 & 15 & 6 & 80423.120 & 0.060 & 3.56 \\
\hline 19 & 16 & 3 & 20 & 16 & 4 & 80411.280 & -0.126 & 6.89 \\
\hline 19 & 16 & 4 & 20 & 16 & 5 & 80411.280 & -0.126 & 6.89 \\
\hline 19 & 17 & 2 & 20 & 17 & 3 & $804 \odot 2.64 \odot$ & 0.093 & 10.44 \\
\hline 19 & 17 & 3 & 20 & 17 & 4 & 80402.640 & 0.093 & 10.44 \\
\hline 21 & 2 & 19 & 21 & 3 & 18 & 30699.720 & -0.036 & -8.13 \\
\hline 21 & 4 & 17 & 21 & 5 & 16 & $30367.30 \odot$ & $-\odot .046$ & 12.31 \\
\hline 21 & 4 & 18 & 21 & 5 & 17 & 40396.780 & 0.084 & 4.96 \\
\hline 21 & 5 & 16 & 21 & 6 & 15 & $44524.89 \odot$ & 0.062 & 11.67 \\
\hline 21 & 6 & 16 & 21 & 7 & 15 & 55831.890 & -0.130 & 7.33 \\
\hline 22 & 1 & 21 & 22 & 2 & 20 & 44740.810 & 0.078 & -2.90 \\
\hline 22 & 1 & 22 & 22 & 2 & 21 & 51533.200 & -0.043 & 2.25 \\
\hline 22 & 3 & 20 & 22 & 4 & 19 & 41269.730 & 0.124 & 0.15 \\
\hline 22 & 5 & 17 & 22 & 6 & 16 & $43399.80 \odot$ & -0.101 & 14.49 \\
\hline 22 & 6 & 16 & 22 & 7 & 15 & 55183.880 & 0.112 & 10.00 \\
\hline 23 & 4 & 20 & 23 & 5 & 19 & 42082.340 & 0.040 & 4.26 \\
\hline 23 & 5 & 18 & 23 & 6 & 17 & 42052.460 & -0.261 & 17.60 \\
\hline 23 & 6 & 17 & 23 & 7 & 16 & 54675.450 & 0.263 & 12.45 \\
\hline 23 & 6 & 18 & 23 & 7 & 17 & 55340.750 & $-\odot .036$ & 10.73 \\
\hline 24 & 2 & 22 & 24 & 3 & 21 & $4 \odot 829.79 \odot$ & $\odot .092$ & -10.76 \\
\hline 24 & 2 & 23 & 24 & 3 & 22 & 50856.380 & 0.234 & -0.44 \\
\hline 24 & 3 & 21 & 24 & 4 & 20 & 28562.950 & $-\odot .268$ & -9.62 \\
\hline 24 & 4 & 20 & 24 & 5 & 19 & $27399.04 \odot$ & 0.113 & 10.48 \\
\hline 24 & 6 & 18 & 24 & 7 & 17 & 54035.950 & 0.087 & 15.34 \\
\hline 24 & 6 & 19 & 24 & 7 & 18 & 55087.680 & 0.078 & 12.45 \\
\hline 25 & $\odot$ & 25 & 25 & 1 & 24 & 59132.820 & -0.216 & 3.76 \\
\hline 25 & 1 & 24 & 25 & 2 & 23 & 53017.250 & $-\odot .169$ & -1.62 \\
\hline 25 & 1 & 25 & 25 & 2 & 24 & 59151.910 & -0.142 & 3.84 \\
\hline 25 & 2 & 23 & 25 & 3 & 22 & 44155.030 & -0.031 & -10.76 \\
\hline 25 & 3 & 22 & 25 & 4 & 21 & 31387.640 & ๑. 086 & -13.55 \\
\hline 25 & 4 & 21 & 25 & 5 & 20 & 27223.060 & $\odot .094$ & 7.60 \\
\hline 25 & 4 & 22 & 25 & 5 & 21 & 44558.130 & 0.069 & 2.55 \\
\hline 25 & 6 & 19 & 25 & 7 & 18 & 53233.980 & 0.134 & 18.76 \\
\hline
\end{tabular}




\begin{tabular}{|c|c|c|c|c|c|c|}
\hline 25 & 620 & 25 & $\begin{array}{ll}7 & 19\end{array}$ & 54845.670 & -0.174 & 14.12 \\
\hline 26 & 125 & 26 & 224 & 55685.270 & $-\odot .062$ & -1.15 \\
\hline 26 & 225 & 26 & 324 & 55935.930 & $-\odot . \odot \odot 9$ & -0.15 \\
\hline 26 & 323 & 26 & 422 & 34581.750 & 0.098 & -17.26 \\
\hline 26 & 521 & 26 & 620 & 37101.940 & -0.042 & 25.90 \\
\hline 26 & 620 & 26 & $7 \quad 19$ & $52236.00 \odot$ & $-\odot . \odot 46$ & 22.81 \\
\hline 26 & 621 & 26 & 720 & 54632.320 & -0.018 & 15.67 \\
\hline 27 & 126 & 27 & 225 & 58324.910 & -0.037 & -0.65 \\
\hline 27 & 225 & 27 & 324 & 50491.470 & -0.061 & -9.91 \\
\hline 27 & 226 & 27 & 325 & 58492.460 & $-\odot .178$ & $\odot .11$ \\
\hline 27 & 324 & 27 & 423 & 38035.410 & 0.214 & -20.40 \\
\hline 27 & 325 & 27 & 424 & 52389.710 & 0.143 & -3.10 \\
\hline 27 & 423 & 27 & 522 & 28546.710 & -0.305 & -1.92 \\
\hline 27 & 22 & 27 & 621 & 35458.320 & 0.081 & 26.87 \\
\hline 27 & 621 & 27 & 720 & 51014.120 & -0.177 & 27.52 \\
\hline 27 & 622 & 27 & 721 & 54467.500 & $\odot .236$ & 17.02 \\
\hline 28 & 127 & 28 & 226 & 60943.550 & 0.154 & -0.13 \\
\hline 28 & 226 & 28 & 325 & 53498.620 & -0.194 & -9.26 \\
\hline 28 & 25 & 28 & 424 & 41632.230 & -0.051 & -22.69 \\
\hline 28 & 26 & 28 & 425 & 54863.020 & $-\odot .099$ & -3.58 \\
\hline 28 & 424 & 28 & 523 & 30107.760 & -0.161 & -8.39 \\
\hline 28 & 523 & 28 & 622 & 34029.380 & $\odot .0 \odot 8$ & 26.20 \\
\hline 29 & 227 & 29 & 326 & 56414.270 & 0.057 & -8.58 \\
\hline 29 & 327 & 29 & 426 & 57380.640 & $-\odot .026$ & -3.96 \\
\hline 29 & 25 & 29 & 524 & 32259.500 & $-\odot .075$ & -15.68 \\
\hline 29 & 26 & 29 & 525 & 51749.930 & -0.018 & -3.07 \\
\hline 29 & 524 & 29 & 623 & 32947.220 & -0.183 & 23.55 \\
\hline 29 & 624 & 29 & 723 & 54376.300 & 0.412 & 18.71 \\
\hline 29 & 722 & 29 & 821 & 61863.880 & 0.174 & 27.96 \\
\hline 30 & 228 & 30 & 327 & 59254.050 & $\odot .257$ & -7.90 \\
\hline 30 & 28 & 30 & 427 & 59929.800 & $-\odot .058$ & -4.22 \\
\hline 30 & 426 & 30 & 525 & 34953.910 & -0.076 & -23.32 \\
\hline 30 & 427 & 30 & 526 & 53930.950 & $-\odot . \odot 86$ & -4.64 \\
\hline 30 & 525 & 30 & 624 & 32328.450 & 0.049 & 18.71 \\
\hline 30 & 625 & 30 & 724 & 54499.640 & 0.044 & 18.86 \\
\hline 30 & 23 & 30 & 822 & 60992.620 & -0.217 & 33.18 \\
\hline 31 & 28 & 31 & 427 & 52345.550 & $\odot .0 \odot 6$ & -24.07 \\
\hline 31 & 27 & 31 & 526 & 38109.480 & 0.016 & -30.69 \\
\hline 31 & 428 & 31 & 527 & 56226.370 & 0.050 & -6.15 \\
\hline 31 & 526 & 31 & 625 & 32264.210 & 0.233 & 11.66 \\
\hline 31 & 25 & 31 & 724 & 44026.730 & $\odot .003$ & 47.67 \\
\hline 31 & 26 & 31 & 725 & 54769.340 & 0.175 & 18.43 \\
\hline 32 & 28 & 32 & 527 & 41616.950 & $-\odot .060$ & -37.18 \\
\hline 32 & 527 & 32 & 626 & 32817.370 & -0.135 & 2.45 \\
\hline 32 & 528 & 32 & 627 & 54169.890 & -0.059 & 0.31 \\
\hline 32 & 626 & 32 & 725 & 42070.680 & $-\odot .0 \odot 8$ & 50.02 \\
\hline 32 & 25 & 32 & 824 & 58637.300 & -0.017 & 46.61 \\
\hline 33 & 330 & 33 & 429 & 58940.300 & -0.291 & -22.06 \\
\hline 33 & 430 & 33 & 529 & 61079.570 & 0.199 & -8.81 \\
\hline 33 & 528 & 33 & 627 & 34022.670 & 0.053 & -8.67 \\
\hline 33 & 529 & 33 & 628 & 55951.850 & 0.307 & -2.49 \\
\hline 33 & 627 & 33 & 726 & 40266.480 & -0.268 & 49.76 \\
\hline 33 & 628 & 33 & 727 & 55831.890 & 0.310 & 15.55 \\
\hline 33 & 726 & 33 & 825 & 57098.790 & -0.218 & 54.80 \\
\hline 34 & 529 & 34 & 628 & 35880.980 & -0.267 & -21.28 \\
\hline 34 & 530 & 34 & 629 & 57905.770 & 0.188 & -5.41 \\
\hline 34 & 628 & 34 & 727 & 38758.990 & $-\odot .050$ & 46.27 \\
\hline
\end{tabular}




\begin{tabular}{|c|c|c|c|c|c|c|c|c|}
\hline 34 & 7 & 28 & 34 & 8 & 27 & 61708.940 & 0.108 & 31.82 \\
\hline 35 & 4 & 31 & 35 & 5 & 30 & 53034.750 & 0.016 & -47.00 \\
\hline 35 & 5 & 31 & 35 & 6 & 30 & 60013.520 & ๑. 055 & -8.36 \\
\hline 35 & 6 & 29 & 35 & 7 & 28 & 37682.860 & 0.108 & 39.21 \\
\hline 35 & 6 & 30 & 35 & 7 & 29 & 57693.470 & 0.152 & 9.89 \\
\hline 35 & 7 & 28 & 35 & 8 & 27 & $53300.60 \odot$ & 0.223 & 72.17 \\
\hline 36 & 4 & 32 & 36 & 5 & 31 & 56795.670 & $\odot .150$ & -46.92 \\
\hline 36 & 5 & 31 & 36 & 6 & 30 & 41392.160 & $-\odot .091$ & -47.93 \\
\hline 36 & 6 & 30 & 36 & 7 & 29 & 37151.980 & 0.118 & 28.45 \\
\hline 36 & 7 & 29 & 36 & 8 & 28 & 51133.220 & $\odot .00 \odot$ & 79.58 \\
\hline 37 & 4 & 33 & 37 & 5 & 32 & 60428.240 & -0.162 & -45.69 \\
\hline 37 & 5 & 32 & 37 & 6 & 31 & 44874.030 & $-\odot .013$ & $-6 \odot .0 \odot$ \\
\hline 37 & 6 & 31 & 37 & 7 & 30 & 37251.710 & $-\odot .189$ & 14.13 \\
\hline 38 & 6 & 32 & 38 & 7 & 31 & 38037.000 & 0.175 & -3.39 \\
\hline 39 & 5 & 34 & 39 & 6 & 33 & 52678.450 & -0.104 & -76.98 \\
\hline 39 & 6 & 33 & 39 & 7 & 32 & 39527.220 & $-\odot .156$ & -23.47 \\
\hline 39 & 7 & 32 & 39 & 8 & 31 & 44859.260 & $-\odot .068$ & 82.18 \\
\hline 40 & 5 & 35 & 40 & 6 & 34 & 56741.040 & $-\odot .005$ & -80.94 \\
\hline 40 & 6 & 34 & 40 & 7 & 33 & 41708.840 & $-\odot .026$ & -45.17 \\
\hline 40 & 7 & 33 & 40 & 8 & 32 & 43327.890 & 0.077 & 73.09 \\
\hline 40 & 8 & 32 & 40 & 9 & 31 & 60718.470 & 0.118 & 111.08 \\
\hline 41 & 6 & 35 & 41 & 7 & 34 & 44528.960 & $-0.04 \odot$ & -67.16 \\
\hline 41 & 7 & 34 & 41 & 8 & 33 & 42311.480 & -0.255 & 58.21 \\
\hline 41 & 8 & 33 & 41 & 9 & 32 & 58404.230 & -0.147 & 123.27 \\
\hline 42 & 8 & 34 & 42 & 9 & 33 & 55970.220 & 0.208 & 132.55 \\
\hline 43 & 6 & 37 & 43 & 7 & 36 & 51697.170 & 0.087 & -105.72 \\
\hline 43 & 8 & 35 & 43 & 9 & 34 & 53537.910 & -0.231 & 136.97 \\
\hline 44 & 6 & 38 & 44 & 7 & 37 & 55785.720 & -0.305 & -119.41 \\
\hline 44 & 7 & 37 & 44 & 8 & 36 & 43285.950 & 0.287 & -17.94 \\
\hline 44 & 8 & 36 & 44 & 9 & 35 & 51257.990 & 0.062 & 134.76 \\
\hline 47 & 7 & 40 & 47 & 8 & 39 & 50780.150 & 0.269 & -118.23 \\
\hline 48 & 7 & 41 & 48 & 8 & 40 & 54482.680 & -0.113 & -148.08 \\
\hline 49 & 7 & 42 & 49 & 8 & 41 & 58584.930 & -0.034 & -172.42 \\
\hline 49 & 9 & 40 & 49 & 10 & 39 & 57950.970 & -0.209 & 207.10 \\
\hline 51 & 8 & 43 & 51 & 9 & 42 & 50732.450 & 0.153 & -96.41 \\
\hline 52 & 8 & 44 & 52 & 9 & 43 & 53606.320 & $-\odot .271$ & -146.61 \\
\hline 53 & 9 & 44 & 53 & 10 & 43 & 51422.860 & -0.013 & 96.93 \\
\hline 54 & 8 & 46 & 54 & 9 & 45 & 61148.450 & -0.065 & -233.78 \\
\hline 54 & 9 & 45 & 54 & 10 & 44 & 51442.860 & -0.175 & 40.67 \\
\hline 56 & 10 & 46 & 56 & 11 & 45 & 59771.050 & -0.169 & 274.91 \\
\hline 57 & 9 & 48 & 57 & 10 & 47 & 56465.800 & 0.165 & -166.46 \\
\hline 57 & 10 & 47 & 57 & 11 & 46 & 57831.820 & $\odot .084$ & 236.19 \\
\hline 58 & 9 & 49 & 58 & 10 & 48 & 59697.520 & 0.079 & -236.23 \\
\hline 58 & 10 & 48 & 58 & 11 & 47 & 56525.200 & 0.068 & 180.36 \\
\hline 59 & 10 & 49 & 59 & 11 & 48 & 55971.640 & -0.044 & 108.90 \\
\hline 61 & 10 & 51 & 61 & 11 & 50 & 57411.270 & $-\odot .021$ & -69.81 \\
\hline 62 & 10 & 52 & 62 & 11 & 51 & 59441.970 & $-\odot .079$ & -169.11 \\
\hline
\end{tabular}

\section{STOPPED ON DIVERGENCY}

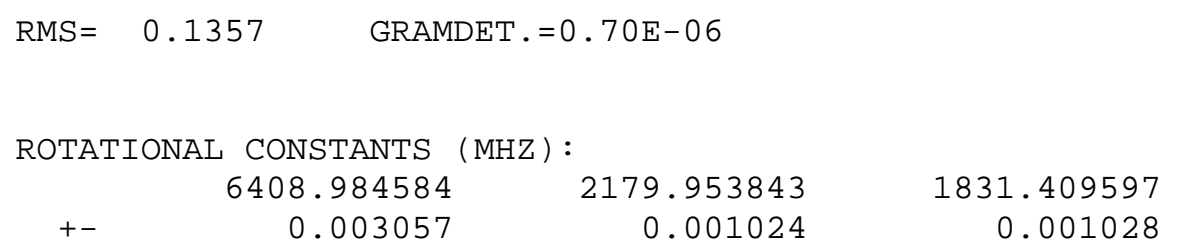




$\begin{array}{rrrrrr}\text { QUARTIC } & \text { DISTORTION } & \text { CONSTANTS: } & & & \\ & 0.64317189 & -2.71226446 & 8.65598628 & 0.03214178 & 0.46640069 \\ +- & 0.00187595 & 0.00232216 & 0.04310062 & 0.00009786 & 0.00440386\end{array}$

\section{SIGNIFICANT DIGITS AND CORRELATION MATRIX:}

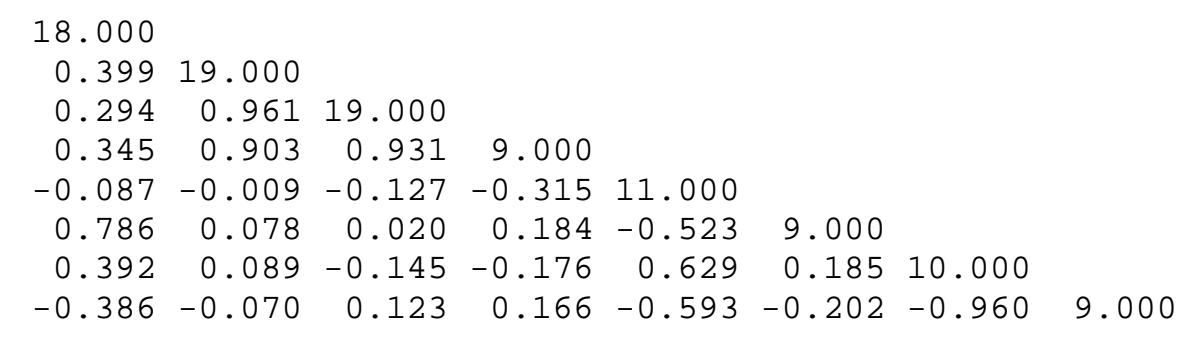

\section{DERIVED CONSTANTS:}

KAPPA $=-\odot .847717$

MOMENTS OF INERTIA AND DEFECT $\left(U^{*} A * * 2\right)$ :

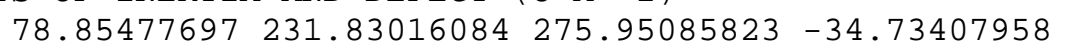

$\begin{array}{lllll}+- & 0.00003761 & 0.00010892 & 0.00015487 & 0.00006847\end{array}$

STANDARD DISTORTION CONSTANTS (KHZ):

$\begin{array}{rrr}-26.347575 & -2.829822 & -2.315553 \\ 0.857671 & -2.572688 & 4.846011\end{array}$


TABLE 2S: Microwave Spectrum of the First Excited State of the Lowest Bending Vibration of Conformer II of Cyclopentadienylphosphine

TOTAL NUMBER OF ACCEPTED TRANSITIONS: 259

\begin{tabular}{|c|c|}
\hline FIXED & CONSTANTS: \\
\hline D $6=$ & $\odot .000 \odot \odot \odot \odot$ \\
\hline D $7=$ & $\odot . \odot \odot \odot \odot \odot \odot \odot$ \\
\hline D $8=$ & $\odot .00000 \odot \odot$ \\
\hline D $9=$ & $\odot .000 \odot \odot \odot \odot$ \\
\hline D10 $=$ & $\odot .000000 \odot$ \\
\hline D11= & $\odot .000000 \odot$ \\
\hline D12= & $\odot .000000 \odot$ \\
\hline RMS $=$ & GRAMDET.$=\odot \cdot 34 \mathrm{E}-06$ \\
\hline
\end{tabular}

TRANSITION

\begin{tabular}{|c|c|c|c|c|c|}
\hline \multicolumn{6}{|c|}{$\mathrm{J}^{\prime \prime}{ }_{K-1}{ }^{\prime}{ }_{K+1}{ }^{\prime} \rightarrow \mathrm{J}^{\prime}{ }_{k-1}{ }^{\prime}{ }_{k+1}^{\prime}$} \\
\hline 6 & 1 & 5 & 7 & 1 & 6 \\
\hline 6 & $\odot$ & 6 & 7 & 1 & 7 \\
\hline 6 & 2 & 4 & 7 & 2 & \\
\hline 6 & 2 & 5 & 7 & 2 & \\
\hline 6 & 3 & 3 & 7 & 3 & \\
\hline 6 & 4 & 3 & 7 & 4 & \\
\hline 6 & 5 & 1 & 7 & 5 & \\
\hline 6 & 5 & 2 & 7 & 5 & \\
\hline 7 & 1 & 6 & 8 & 2 & \\
\hline 7 & 3 & 4 & 8 & 3 & \\
\hline 7 & 4 & 3 & 8 & 4 & \\
\hline 7 & 6 & 1 & 8 & 6 & \\
\hline 7 & 6 & 2 & 8 & 6 & \\
\hline 7 & 7 & $\odot$ & 8 & 7 & \\
\hline 7 & 7 & 1 & 8 & 7 & \\
\hline 8 & 1 & 8 & 9 & 0 & \\
\hline 8 & $\odot$ & 8 & 9 & 1 & \\
\hline 8 & 3 & 5 & 9 & 3 & \\
\hline 8 & 3 & 6 & 9 & 3 & \\
\hline 8 & 4 & 4 & 9 & 4 & \\
\hline 8 & 4 & 5 & 9 & 4 & \\
\hline 9 & 3 & 7 & 9 & 4 & \\
\hline 9 & 6 & 3 & 9 & 7 & \\
\hline 9 & 6 & 4 & 9 & 7 & \\
\hline 8 & 8 & $\odot$ & 9 & 8 & \\
\hline 8 & 8 & 1 & 9 & 8 & \\
\hline 9 & 1 & 8 & 10 & 1 & \\
\hline 9 & 3 & 6 & 10 & 3 & \\
\hline 9 & 2 & 7 & 10 & 3 & \\
\hline 9 & 3 & 7 & 10 & 3 & \\
\hline 9 & 4 & 5 & 10 & 4 & \\
\hline 10 & 6 & 4 & 10 & 7 & \\
\hline 10 & 6 & 5 & 10 & 7 & \\
\hline 10 & 4 & 6 & 11 & 4 & \\
\hline 16 & 4 & 7 & 11 & 4 & \\
\hline 10 & 5 & 5 & 11 & 5 & \\
\hline 0 & 5 & 6 & 11 & 5 & \\
\hline
\end{tabular}

OBS. FREQ. O. C. TOTAL

\begin{tabular}{|c|c|c|}
\hline 28975.580 & $-\odot . \odot \odot 7$ & -0.83 \\
\hline 28454.310 & $-\odot .058$ & -0.63 \\
\hline 28888.170 & $-\odot .0 \odot 6$ & -0.78 \\
\hline 27917.550 & -0.110 & -0.69 \\
\hline 28296.940 & -0.013 & -0.56 \\
\hline 28178.730 & $-\odot . \odot \odot 8$ & $-\odot .31$ \\
\hline 28150.730 & $-\odot . \odot 98$ & $\odot .01$ \\
\hline 28150.730 & $-\odot .062$ & 0.01 \\
\hline 40531.290 & 0.575 & -0.68 \\
\hline 32430.430 & -0.102 & -0.96 \\
\hline 32236.940 & -0.261 & -0.66 \\
\hline 32166.660 & 0.043 & 0.14 \\
\hline 32166.660 & $\odot .044$ & 0.14 \\
\hline 32153.390 & -0.108 & 0.66 \\
\hline 32153.390 & -0.108 & 0.66 \\
\hline 33418.760 & 0.296 & -1.74 \\
\hline $35 \odot 56.570$ & -0.198 & -1.46 \\
\hline 36611.610 & 0.304 & -1.50 \\
\hline 36288.860 & $-\odot .078$ & -1.42 \\
\hline 36307.070 & 0.040 & -1.13 \\
\hline 36288.860 & -0.053 & -1.12 \\
\hline 30717.170 & -0.191 & $\odot .15$ \\
\hline 57177.160 & 0.133 & -5.90 \\
\hline 57177.160 & 0.125 & -5.90 \\
\hline 36171.320 & -0.176 & 1.02 \\
\hline 36171.320 & $-\odot .176$ & 1.02 \\
\hline 40749.250 & $-\odot .199$ & -2.32 \\
\hline 40845.800 & 0.158 & -2.20 \\
\hline 58620.790 & $-\odot .152$ & -1.11 \\
\hline 40320.570 & $-\odot .025$ & -2.05 \\
\hline 40394 . 840 & -0.151 & -1.75 \\
\hline 57151.090 & 0.117 & -5.26 \\
\hline $57151.09 \odot$ & $\odot . \odot 87$ & -5.26 \\
\hline 44506.270 & $-\odot .056$ & -2.53 \\
\hline 44430.380 & $-\odot .038$ & -2.49 \\
\hline 44350.880 & $-\odot .190$ & -1.99 \\
\hline 44348.140 & 0.255 & -1.99 \\
\hline
\end{tabular}




\begin{tabular}{|c|c|c|c|c|c|c|c|c|}
\hline 12 & 3 & 9 & 13 & 3 & 10 & 53816.770 & 0.245 & -5.40 \\
\hline 12 & 4 & 8 & 13 & 4 & 9 & $52827.04 \odot$ & ๑. 091 & -4.69 \\
\hline 12 & 4 & 9 & 13 & 4 & 10 & 52589.070 & -0.037 & -4.53 \\
\hline 12 & 5 & 8 & 13 & 5 & 9 & 52495.910 & $-\odot .016$ & -3.95 \\
\hline 13 & 4 & 10 & 13 & 5 & 9 & 39126.680 & 0.045 & 1.16 \\
\hline 12 & 7 & 5 & 13 & 7 & 6 & $52344.09 \odot$ & 0.010 & -2.36 \\
\hline 12 & 7 & 6 & 13 & 7 & 7 & 52344.090 & 0.021 & -2.36 \\
\hline 12 & 8 & 4 & 13 & 8 & 5 & 52307.280 & $-\odot .099$ & -1.39 \\
\hline 12 & 8 & 5 & 13 & 8 & 6 & 52307.280 & $-\odot .099$ & -1.39 \\
\hline 12 & 9 & 3 & 13 & 9 & 4 & 52283.070 & 0.138 & -0.29 \\
\hline 12 & 9 & 4 & 13 & 9 & 5 & 52283.070 & 0.138 & -0.29 \\
\hline 12 & 10 & 2 & 13 & 10 & 3 & 52266.200 & 0.189 & 0.93 \\
\hline 12 & 10 & 3 & 13 & 10 & 4 & $52266.20 \odot$ & 0.189 & 0.93 \\
\hline 12 & 11 & 1 & 13 & 11 & 2 & 52253.780 & -0.221 & 2.28 \\
\hline 12 & 11 & 2 & 13 & 11 & 3 & 52253.780 & -0.221 & 2.28 \\
\hline 12 & 12 & 0 & 13 & 12 & 1 & $52245.06 \odot$ & -0.291 & 3.76 \\
\hline 12 & 12 & 1 & 13 & 12 & 2 & $52245.06 \odot$ & -0.291 & 3.76 \\
\hline 13 & 1 & 13 & 14 & 0 & 14 & 52441.850 & -0.101 & -6.11 \\
\hline 13 & 2 & 12 & 14 & 1 & 13 & 53058.970 & 0.184 & -7.02 \\
\hline 13 & 0 & 13 & 14 & 1 & 14 & 52652.250 & 0.052 & -5.97 \\
\hline 13 & 3 & 10 & 14 & 3 & 11 & 58165.650 & 0.022 & -6.84 \\
\hline 14 & 2 & 13 & 14 & 3 & 12 & $29051.44 \odot$ & 0.240 & 0.61 \\
\hline 13 & 4 & 9 & 14 & 4 & 10 & 57052.370 & 0.103 & -6.11 \\
\hline 14 & 3 & 12 & 14 & 4 & 11 & 31697.130 & $-\odot . \odot 9 \odot$ & 1.63 \\
\hline 13 & 5 & 8 & 14 & 5 & 9 & 56612.870 & $-\odot .075$ & -5.26 \\
\hline 14 & 4 & 10 & 14 & 5 & 9 & 38194.110 & 0.020 & 2.29 \\
\hline 14 & 4 & 11 & 14 & 5 & 10 & 39043.390 & -0.056 & 1.76 \\
\hline 13 & 7 & 6 & 14 & 7 & 7 & $56396.97 \odot$ & 0.061 & -3.50 \\
\hline 14 & 6 & 8 & 14 & 7 & 7 & 56937.920 & -0.028 & -1.98 \\
\hline 13 & 7 & 7 & 14 & 7 & 8 & 56396.970 & 0.094 & -3.50 \\
\hline 13 & 8 & 5 & 14 & 8 & 6 & 56350.900 & 0.113 & -2.45 \\
\hline 13 & 8 & 6 & 14 & 8 & 7 & 56350.900 & 0.114 & -2.45 \\
\hline 13 & 9 & 4 & 14 & 9 & 5 & 56320.150 & 0.123 & -1.26 \\
\hline 13 & 9 & 5 & 14 & 9 & 6 & 56320.150 & 0.123 & -1.26 \\
\hline 13 & 10 & 3 & 14 & 10 & 4 & 56298.550 & -0.118 & 0.06 \\
\hline 13 & 10 & 4 & 14 & 10 & 5 & 56298.550 & -0.118 & 0.06 \\
\hline 13 & 11 & 2 & 14 & 11 & 3 & 56283.370 & -0.060 & 1.51 \\
\hline 13 & 11 & 3 & 14 & 11 & 4 & 56283.370 & -0.060 & 1.51 \\
\hline 13 & 12 & 1 & 14 & 12 & 2 & 56272.550 & 0.180 & 3.10 \\
\hline 13 & 12 & 2 & 14 & 12 & 3 & 56272.550 & 0.180 & 3.10 \\
\hline 14 & $\odot$ & 14 & 15 & $\odot$ & 15 & 56222.540 & 0.076 & -7.43 \\
\hline 14 & 1 & 14 & 15 & $\odot$ & 15 & 56140.340 & -0.101 & -7.49 \\
\hline 14 & 1 & 13 & 15 & 1 & 14 & 59054.230 & 0.136 & -7.30 \\
\hline 14 & $\odot$ & 14 & 15 & 1 & 15 & 56274.420 & $-\odot .044$ & -7.38 \\
\hline 14 & 1 & 14 & 15 & 1 & 15 & 56192.340 & -0.101 & -7.44 \\
\hline 15 & 2 & 14 & 15 & 3 & 13 & 30685.960 & 0.281 & 0.51 \\
\hline 14 & 4 & 10 & 15 & 4 & 11 & 61330.270 & 0.109 & -7.81 \\
\hline 14 & 4 & 11 & 15 & 4 & 12 & 60736.340 & $-\odot .085$ & -7.33 \\
\hline 14 & 5 & 9 & 15 & 5 & 10 & 60733.900 & -0.063 & -6.78 \\
\hline 15 & 4 & 11 & 15 & 5 & 10 & 37597.640 & -0.252 & 3.31 \\
\hline 14 & 5 & 10 & 15 & 5 & 11 & 60678.870 & $\odot .072$ & -6.72 \\
\hline 15 & 4 & 12 & 15 & 5 & 11 & 38985.770 & -0.049 & 2.37 \\
\hline 14 & 6 & 8 & 15 & 6 & 9 & 60547.630 & -0.264 & -5.86 \\
\hline 14 & 7 & 7 & 15 & 7 & 8 & 60455.710 & $-\odot .0 \odot 4$ & -4.86 \\
\hline 15 & 6 & 9 & 15 & 7 & 8 & 56845.890 & 0.122 & -0.98 \\
\hline 14 & 7 & 8 & 15 & 7 & 9 & 60455.710 & ๑.๑८० & -4.86 \\
\hline 14 & 8 & 6 & 15 & 8 & 7 & 60398.430 & -0.198 & -3.72 \\
\hline
\end{tabular}




\begin{tabular}{|c|c|c|c|c|c|c|c|c|}
\hline 14 & 8 & 7 & 15 & 8 & 8 & 60398.430 & -0.196 & -3.72 \\
\hline 14 & 9 & 5 & 15 & 9 & 6 & 60360.370 & -0.176 & -2.45 \\
\hline 14 & 9 & 6 & 15 & 9 & 7 & 60360.370 & -0.176 & -2.45 \\
\hline 14 & 10 & 4 & 15 & 10 & 5 & 60334.150 & 0.107 & -1.03 \\
\hline 14 & 10 & 5 & 15 & 10 & 6 & 60334.150 & 0.107 & -1.03 \\
\hline$L 4$ & 11 & 3 & 15 & 11 & 4 & 60315.290 & 0.230 & 0.53 \\
\hline 14 & 11 & 4 & 15 & 11 & 5 & 60315.290 & 0.230 & 0.53 \\
\hline 14 & 13 & 1 & 15 & 13 & 2 & $6 \odot 291.040$ & 0.066 & 4.09 \\
\hline 14 & 13 & 2 & 15 & 13 & 3 & $6 \odot 291.040$ & 0.066 & 4.09 \\
\hline 15 & 0 & 15 & 16 & 0 & 16 & 59877.470 & -0.065 & -9.03 \\
\hline 15 & 2 & 14 & 16 & 1 & 15 & 61403.820 & $-\odot .029$ & -9.76 \\
\hline 15 & 0 & 15 & 16 & 1 & 16 & 59910.410 & 0.159 & -8.99 \\
\hline 16 & 2 & 15 & 16 & 3 & 14 & 32488.060 & ๑. 083 & 0.36 \\
\hline 16 & 4 & 12 & 16 & 5 & 11 & 36814.450 & 0.190 & 4.53 \\
\hline 16 & 4 & 13 & 16 & 5 & 12 & 38973.210 & 0.118 & 2.96 \\
\hline 15 & 6 & 10 & 16 & 6 & 11 & 64629.310 & -0.031 & -7.53 \\
\hline 15 & 7 & 8 & 16 & 7 & 9 & $64521.00 \odot$ & $-\odot .003$ & -6.45 \\
\hline 16 & 6 & 10 & 16 & 7 & 9 & 56731.730 & -0.035 & 0.11 \\
\hline 15 & 7 & 9 & 16 & 7 & 10 & 64521.000 & 0.202 & -6.45 \\
\hline 15 & 8 & 7 & 16 & 8 & 8 & 64451.180 & $-\odot .064$ & -5.23 \\
\hline 15 & 8 & 8 & 16 & 8 & 9 & 64451.180 & $-\odot .059$ & -5.23 \\
\hline 15 & 9 & 6 & 16 & 9 & 7 & 64404.700 & -0.044 & -3.86 \\
\hline 15 & 9 & 7 & 16 & 9 & 8 & 64404.700 & -0.044 & -3.86 \\
\hline 15 & 10 & 5 & 16 & 10 & 6 & 64372.200 & -0.134 & -2.35 \\
\hline 15 & 10 & 6 & 16 & 10 & 7 & 64372.200 & -0.134 & -2.35 \\
\hline 15 & 11 & 4 & 16 & 11 & 5 & 64349.000 & -0.051 & -0.68 \\
\hline 15 & 11 & 5 & 16 & 11 & 6 & 64349.000 & -0.051 & -0.68 \\
\hline 15 & 12 & 3 & 16 & 12 & 4 & 64332.290 & 0.316 & 1.14 \\
\hline 15 & 12 & 4 & 16 & 12 & 5 & 64332.290 & 0.316 & 1.14 \\
\hline 17 & 1 & 16 & 17 & 2 & 15 & 29445.440 & -0.106 & -3.29 \\
\hline 16 & 4 & 13 & 17 & 4 & 14 & 68833.840 & -0.127 & -10.96 \\
\hline 16 & 5 & 11 & 17 & 5 & 12 & 69053.340 & -0.109 & -10.64 \\
\hline 16 & 5 & 12 & 17 & 5 & 13 & 68888.440 & $-\odot .067$ & -10.42 \\
\hline 17 & 4 & 14 & 17 & 5 & 13 & 39027.520 & -0.112 & 3.51 \\
\hline 16 & 6 & 10 & 17 & 6 & 11 & 68733.840 & 0.259 & -9.47 \\
\hline 16 & 6 & 11 & 17 & 6 & 12 & 68722.450 & 0.015 & -9.45 \\
\hline 17 & 6 & 11 & 17 & 7 & 10 & 56591.540 & 0.030 & 1.29 \\
\hline 16 & 8 & 8 & 17 & 8 & 9 & 68508.910 & $-\odot .079$ & -6.98 \\
\hline 16 & 8 & 9 & 17 & 8 & 10 & 68508.910 & -0.066 & -6.98 \\
\hline 16 & 9 & 7 & 17 & 9 & 8 & 68452.940 & 0.057 & -5.52 \\
\hline 16 & 9 & 8 & 17 & 9 & 9 & 68452.940 & 0.058 & -5.52 \\
\hline 16 & 10 & 6 & 17 & 10 & 7 & 68413.630 & -0.114 & -3.91 \\
\hline 16 & 10 & 7 & 17 & 10 & 8 & 68413.630 & -0.114 & -3.91 \\
\hline 16 & 11 & 5 & 17 & 11 & 6 & 68385.720 & $\odot .156$ & -2.13 \\
\hline 16 & 11 & 6 & 17 & 11 & 7 & 68385.720 & 0.156 & -2.13 \\
\hline 16 & 14 & 2 & 17 & 14 & 3 & 68337.770 & 0.103 & 4.18 \\
\hline 16 & 14 & 3 & 17 & 14 & 4 & 68337.770 & 0.103 & 4.18 \\
\hline 18 & 2 & 17 & 18 & 3 & 16 & 36530.620 & -0.091 & -0.02 \\
\hline 18 & 4 & 15 & 18 & 5 & 14 & 39173.570 & -0.131 & 3.97 \\
\hline 18 & 6 & 12 & 18 & 7 & 11 & 56419.160 & -0.146 & 2.57 \\
\hline 18 & 6 & 13 & 18 & 7 & 12 & 56460.140 & 0.068 & 2.50 \\
\hline 17 & 8 & 9 & 18 & 8 & 10 & 72572.300 & 0.072 & -9.00 \\
\hline 17 & 8 & 10 & 18 & 8 & 11 & 72572.300 & 0.104 & -9.00 \\
\hline 17 & 9 & 8 & 18 & 9 & 9 & 72505.130 & $-\odot .097$ & -7.44 \\
\hline 17 & 9 & 9 & 18 & 9 & 10 & 72505.130 & $-\odot .096$ & -7.44 \\
\hline 17 & 10 & 7 & 18 & 10 & 8 & 72458.460 & -0.017 & -5.73 \\
\hline 17 & 10 & 8 & 18 & 10 & 9 & 72458.460 & -0.017 & -5.73 \\
\hline
\end{tabular}




\begin{tabular}{|c|c|c|c|c|c|c|c|c|}
\hline 17 & 11 & 6 & 18 & 11 & 7 & 72424.720 & -0.041 & -3.84 \\
\hline 17 & 11 & 7 & 18 & 11 & 8 & 72424.720 & -0.041 & -3.84 \\
\hline 17 & 12 & 5 & 18 & 12 & 6 & 72399.920 & 0.048 & -1.78 \\
\hline 17 & 12 & 6 & 18 & 12 & 7 & 72399.920 & $\odot .048$ & -1.78 \\
\hline 19 & 3 & 17 & 19 & 4 & 16 & 36265.300 & $\odot . \odot 29$ & 1.38 \\
\hline 19 & 4 & 15 & 19 & 5 & 14 & 33282.170 & -0.193 & 9.10 \\
\hline 19 & 4 & 16 & 19 & 5 & 15 & 39436.430 & 0.369 & 4.30 \\
\hline 18 & 7 & 11 & 19 & 7 & 12 & 76761.500 & -0.127 & -12.79 \\
\hline 18 & 9 & 9 & 19 & 9 & 10 & 76561.910 & -0.139 & -9.64 \\
\hline 18 & 9 & 10 & 19 & 9 & 11 & 76561.910 & -0.137 & -9.64 \\
\hline 8 & 14 & 4 & 19 & 14 & 5 & 76398.100 & -0.005 & 1.25 \\
\hline 18 & 14 & 5 & 19 & 14 & 6 & 76398.100 & -0.005 & 1.25 \\
\hline 20 & 6 & 14 & 20 & 7 & 13 & 55946.650 & $-\odot .036$ & 5.52 \\
\hline 21 & 3 & 19 & 21 & 4 & 18 & 39411.560 & -0.010 & 0.45 \\
\hline 21 & 4 & 17 & 21 & 5 & 16 & 30410.160 & -0.119 & 11.52 \\
\hline 21 & 4 & 18 & 21 & 5 & 17 & $40402.10 \odot$ & -0.040 & 4.45 \\
\hline 1 & 5 & 16 & 21 & 6 & 15 & 44566.690 & 0.021 & 10.84 \\
\hline 22 & 1 & 21 & 22 & 2 & 20 & 44698.620 & 0.073 & -3.10 \\
\hline 22 & 2 & 20 & 22 & 3 & 19 & 33971.890 & -0.159 & -9.25 \\
\hline 22 & 3 & 20 & 22 & 4 & 19 & 41246.590 & -0.297 & -0.17 \\
\hline 22 & 4 & 18 & 22 & 5 & 17 & 29115.400 & -0.188 & 11.88 \\
\hline 22 & 5 & 17 & 22 & 6 & 16 & 43446.790 & -0.144 & 13.49 \\
\hline 22 & 6 & 16 & 22 & 7 & 15 & 55219.550 & 0.004 & 9.22 \\
\hline 23 & 2 & 21 & 23 & 3 & 20 & 37374.590 & -0.016 & -10.15 \\
\hline 23 & 3 & 20 & 23 & 4 & 19 & 26163.550 & 0.051 & -5.73 \\
\hline 23 & 6 & 18 & 23 & 7 & 17 & 55372.350 & -0.192 & 9.87 \\
\hline 24 & 2 & 22 & 24 & 3 & 21 & 40768.710 & 0.049 & -10.62 \\
\hline 24 & 2 & 23 & 24 & 3 & 22 & 50819.040 & 0.222 & -0.86 \\
\hline 24 & 3 & 21 & 24 & 4 & 20 & 28515.040 & -0.203 & -9.37 \\
\hline 24 & 5 & 19 & 24 & 6 & 18 & 40564.850 & -0.104 & 19.43 \\
\hline 24 & 6 & 18 & 24 & 7 & 17 & 54077.950 & $\odot .091$ & 14.20 \\
\hline 24 & 6 & 19 & 24 & 7 & 18 & 55119.070 & -0.206 & 11.46 \\
\hline 25 & 1 & 24 & 25 & 2 & 23 & 52974.450 & -0.037 & -2.05 \\
\hline 25 & 2 & 23 & 25 & 3 & 22 & 44094.200 & -0.196 & -10.69 \\
\hline 25 & 2 & 24 & 25 & 3 & 23 & 53349.730 & 0.334 & -0.82 \\
\hline 25 & 3 & 22 & 25 & 4 & 21 & 31328.020 & $-\odot .024$ & -13.16 \\
\hline 25 & 4 & 21 & 25 & 5 & 20 & 27233.490 & $-\odot .267$ & 7.03 \\
\hline 25 & 4 & 22 & 25 & 5 & 21 & 44543.410 & 0.041 & 2.01 \\
\hline 25 & 6 & 20 & 25 & 7 & 19 & 54877.240 & 0.177 & 13.00 \\
\hline 26 & 2 & 25 & 26 & 3 & 24 & 55894.820 & -0.169 & -0.71 \\
\hline 26 & 3 & 23 & 26 & 4 & 22 & 34512.550 & -0.017 & -16.76 \\
\hline 26 & 4 & 22 & 26 & 5 & 21 & 27587.160 & -0.126 & 3.08 \\
\hline 26 & 5 & 21 & 26 & 6 & 20 & 37162.380 & -0.066 & 24.27 \\
\hline 26 & 6 & 21 & 26 & 7 & 20 & 54662.660 & $\odot .053$ & 14.43 \\
\hline 27 & 2 & 25 & 27 & 3 & 24 & 50432.930 & $-\odot .088$ & -10.01 \\
\hline 27 & 4 & 23 & 27 & 5 & 22 & 28527.600 & 0.255 & -2.05 \\
\hline 27 & 5 & 22 & 27 & 6 & 21 & 35515.500 & -0.175 & 25.21 \\
\hline 27 & 6 & 21 & 27 & 7 & 20 & 51072.790 & -0.135 & 25.63 \\
\hline 27 & 6 & 22 & 27 & 7 & 21 & 54495.820 & -0.141 & 15.66 \\
\hline 28 & 2 & 26 & 28 & 3 & 25 & 53441.400 & 0.079 & -9.47 \\
\hline 28 & 3 & 25 & 28 & 4 & 24 & 41551.620 & -0.299 & -22.11 \\
\hline 28 & 3 & 26 & 28 & 4 & 25 & 54818.700 & -0.184 & -4.06 \\
\hline 28 & 5 & 23 & 28 & 6 & 22 & 34079.860 & $-\odot .049$ & 24.59 \\
\hline 29 & 3 & 27 & 29 & 4 & 26 & 57333.950 & -0.010 & -4.50 \\
\hline 29 & 4 & 25 & 29 & 5 & 24 & 32206.060 & 0.085 & -15.23 \\
\hline 29 & 4 & 26 & 29 & 5 & 25 & 51713.390 & 0.076 & -3.58 \\
\hline 29 & 7 & 22 & 29 & 8 & 21 & 61914.100 & $-\odot .0 \odot 7$ & 25.90 \\
\hline
\end{tabular}




\begin{tabular}{|c|c|c|c|c|c|c|c|c|}
\hline 30 & 2 & 28 & 30 & 3 & 27 & 59197.590 & 0.210 & -8.34 \\
\hline 30 & 4 & 26 & 30 & 5 & 25 & 34884.500 & ๑. 028 & -22.56 \\
\hline 30 & 6 & 25 & 30 & 7 & 24 & 54518.940 & 0.103 & 17.25 \\
\hline 30 & 7 & 23 & 30 & 8 & 22 & 61048.270 & $-\odot .246$ & 30.79 \\
\hline 31 & 4 & 27 & 31 & 5 & 26 & 38026.190 & $\odot .001$ & -29.68 \\
\hline 31 & 4 & 28 & 31 & 5 & 27 & 56180.420 & $-\odot .076$ & -6.66 \\
\hline 31 & 5 & 26 & 31 & 6 & 25 & 32271.840 & $-\odot .0 \odot 6$ & 10.80 \\
\hline 31 & 6 & 26 & 31 & 7 & 25 & 54783.770 & 0.275 & 16.78 \\
\hline 31 & 7 & 24 & 31 & 8 & 23 & 59990.230 & 0.117 & 36.60 \\
\hline 32 & 3 & 29 & 32 & 4 & 28 & 55630.500 & $-\odot .0 \odot 6$ & -23.02 \\
\hline 32 & 4 & 29 & 32 & 5 & 28 & $58565.84 \odot$ & $\odot .117$ & -8.09 \\
\hline 32 & 5 & 27 & 32 & 6 & 26 & 32805.710 & 0.112 & 2.04 \\
\hline 32 & 5 & 28 & 32 & 6 & 27 & $54142.07 \odot$ & 0.275 & -0.53 \\
\hline 32 & 7 & 25 & 32 & 8 & 24 & 58707.120 & -0.067 & 43.41 \\
\hline 33 & 3 & 30 & 33 & 4 & 29 & $58864.89 \odot$ & 0.006 & -22.04 \\
\hline 33 & 5 & 28 & 33 & 6 & 27 & 33989.380 & -0.079 & -8.57 \\
\hline 33 & 6 & 27 & 33 & 7 & 26 & $40338.02 \odot$ & -0.282 & 46.78 \\
\hline 33 & 6 & 28 & 33 & 7 & 27 & $55833.90 \odot$ & 0.322 & 13.92 \\
\hline 33 & 7 & 27 & 33 & 8 & 26 & 61861.460 & 0.094 & 28.52 \\
\hline 34 & 5 & 29 & 34 & 6 & 28 & 35826.200 & -0.193 & -20.61 \\
\hline 34 & 6 & 28 & 34 & 7 & 27 & 38819.290 & -0.169 & 43.53 \\
\hline 34 & 7 & 27 & 34 & 8 & 26 & $55397.30 \odot$ & -0.188 & 59.42 \\
\hline 35 & 4 & 31 & 35 & 5 & 30 & 52929.320 & -0.143 & -45.80 \\
\hline 35 & 6 & 29 & 35 & 7 & 28 & 37727.250 & $-\odot .074$ & 36.87 \\
\hline 35 & 7 & 28 & 35 & 8 & 27 & 53394.480 & 0.066 & 67.59 \\
\hline 36 & 4 & 32 & 36 & 5 & 31 & 56691.870 & -0.086 & -45.90 \\
\hline 36 & 7 & 29 & 36 & 8 & 28 & $51232.04 \odot$ & $\odot .119$ & 74.67 \\
\hline 37 & 4 & 33 & 37 & 5 & 32 & $60327.90 \odot$ & 0.040 & -44.89 \\
\hline 37 & 6 & 31 & 37 & 7 & 30 & 37253.480 & 0.226 & 13.10 \\
\hline 39 & 5 & 34 & 39 & 6 & 33 & 52550.620 & 0.010 & -74.49 \\
\hline 39 & 7 & 32 & 39 & 8 & 31 & 44943.640 & $-\odot .054$ & 77.45 \\
\hline 40 & 5 & 35 & $4 \odot$ & 6 & 34 & 56610.620 & $-\odot .031$ & -78.51 \\
\hline 40 & 7 & 33 & 40 & 8 & 32 & 43395.880 & -0.018 & 68.94 \\
\hline 41 & 5 & 36 & 41 & 6 & 35 & $60634.84 \odot$ & -0.142 & -79.77 \\
\hline 41 & 7 & 34 & 41 & 8 & 33 & 42358.210 & 0.052 & 54.91 \\
\hline 41 & 8 & 33 & 41 & 9 & 32 & 58522.140 & 0.188 & 115.65 \\
\hline 42 & 8 & 34 & 42 & 9 & 33 & 56091.270 & 0.203 & 124.58 \\
\hline 43 & 6 & 37 & 43 & 7 & 36 & 51557.900 & $\odot .026$ & -101.77 \\
\hline 43 & 8 & 35 & 43 & 9 & 34 & $53657.44 \odot$ & 0.223 & 128.96 \\
\hline 44 & 7 & 37 & 44 & 8 & 36 & $43245.27 \odot$ & 0.221 & -17.35 \\
\hline 44 & 8 & 36 & 44 & 9 & 35 & 51368.550 & $\odot .074$ & 127.09 \\
\hline 47 & 7 & 40 & 47 & 8 & 39 & $50649.30 \odot$ & 0.111 & -113.32 \\
\hline 48 & 7 & 41 & 48 & 8 & 40 & 54329.200 & -0.108 & -142.12 \\
\hline 48 & 9 & 39 & 48 & 10 & 38 & 60696.950 & $-\odot .055$ & 193.58 \\
\hline 49 & 9 & 40 & 49 & 10 & 39 & 58088.690 & -0.249 & 195.34 \\
\hline 52 & 9 & 43 & 52 & 10 & 42 & 52247.320 & 0.228 & 135.39 \\
\hline 53 & 9 & 44 & 53 & 10 & 43 & 51461.750 & -0.015 & 92.24 \\
\hline 54 & 8 & 46 & 54 & 9 & 45 & $60962.19 \odot$ & $-\odot .044$ & -224.17 \\
\hline 54 & 9 & 45 & 54 & 10 & 44 & 51442.320 & 0.021 & 38.96 \\
\hline 57 & 10 & 47 & 57 & 11 & 46 & 57938.570 & -0.283 & 224.27 \\
\hline 58 & 10 & 48 & 58 & 11 & 47 & 56596.220 & $\odot .009$ & 171.81 \\
\hline
\end{tabular}

\section{STOPPED ON DIVERGENCY}

RMS $=0.1481 \quad$ GRAMDET.$=0.34 \mathrm{E}-06$ 


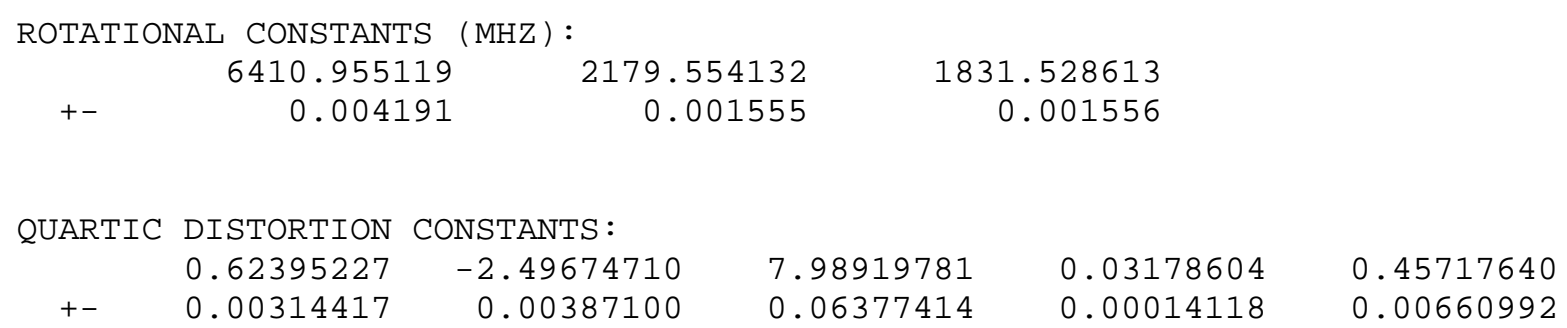

SIGNIFICANT DIGITS AND CORRELATION MATRIX:

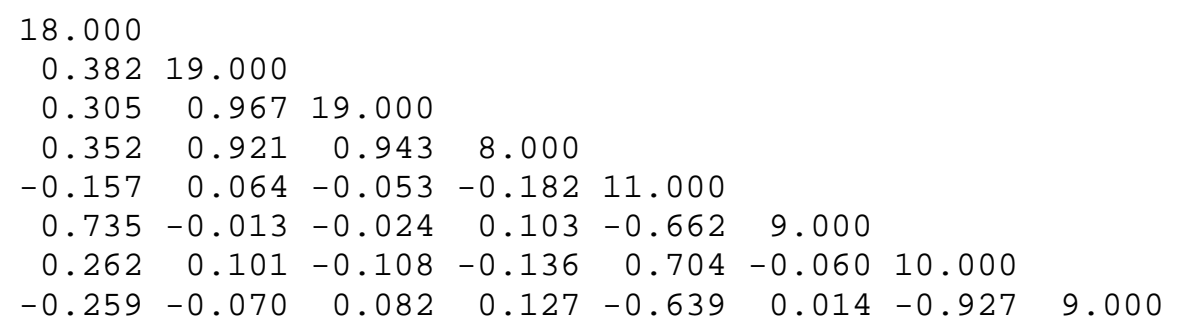

$\begin{array}{rrr}-24.465612 & -2.750097 & -2.241521 \\ 0.541835 & -2.495809 & 4.453535\end{array}$


TABLE 3S: Microwave Spectrum of the Ground Vibrational State of Conformer I of Cyclopentadienylphosphine

\author{
ACCURACY: $\pm 0.15 \mathrm{MHZ}$ \\ TOTAL NUMBER OF ACCEPTED TRANSITIONS: 200 \\ FIXED CONSTANTS: \\ D $6=0.0000000$ \\ D $7=0.0000000$ \\ D $8=0.0000000$ \\ D $9=0.0000000$ \\ $\mathrm{D} 11=\quad 0.0000000$ \\ $\mathrm{D} 12=\quad 0.0000000$ \\ RMS $=0.1638 \quad$ GRAMDET.$=0.31 \mathrm{E}-12$
}

TRANSITION

\begin{tabular}{|c|c|c|c|c|c|}
\hline \multicolumn{6}{|c|}{$\mathrm{J}^{\prime \prime}{ }_{K-1}{ }^{\prime}{ }_{K+1}^{\prime \prime} \rightarrow \mathrm{J}_{K-1}^{\prime}{ }_{K+1}^{\prime}$} \\
\hline 3 & 2 & & 4 & & 2 \\
\hline 4 & 2 & 2 & 5 & 3 & 2 \\
\hline 4 & 2 & 3 & 5 & 3 & \\
\hline 4 & 4 & $\odot$ & 5 & 5 & \\
\hline 4 & 4 & 1 & 5 & 5 & \\
\hline 5 & 1 & 4 & 6 & 2 & \\
\hline 5 & 3 & 2 & 6 & & 2 \\
\hline 5 & 3 & 3 & 6 & 4 & 3 \\
\hline 6 & 0 & 6 & 7 & 1 & \\
\hline 6 & 2 & 5 & 7 & 3 & \\
\hline 6 & 3 & 3 & 7 & 4 & \\
\hline 6 & 3 & 4 & 7 & 4 & \\
\hline 7 & 1 & 6 & 8 & 2 & \\
\hline 7 & 1 & 7 & 8 & 2 & 7 \\
\hline 7 & 2 & 6 & 8 & 3 & 6 \\
\hline 8 & 3 & 6 & 8 & 4 & 4 \\
\hline 8 & 3 & 5 & 8 & 4 & \\
\hline 8 & 6 & 3 & 8 & 7 & $\perp$ \\
\hline 8 & 6 & 2 & 8 & 7 & 2 \\
\hline 8 & 2 & 6 & 9 & 3 & 6 \\
\hline 8 & 2 & 7 & 9 & 3 & 7 \\
\hline 9 & 4 & 6 & 9 & 5 & \\
\hline 9 & 6 & 4 & 9 & 7 & 2 \\
\hline 9 & 6 & 3 & 9 & 7 & 3 \\
\hline 9 & $\odot$ & 9 & 10 & 1 & 9 \\
\hline 9 & 1 & 8 & 10 & 2 & 8 \\
\hline 10 & 3 & 8 & 10 & 4 & \\
\hline 10 & 6 & 5 & 10 & 7 & \\
\hline 10 & 6 & 4 & 10 & 7 & \\
\hline 11 & 3 & 9 & 11 & 4 & 7 \\
\hline 11 & 6 & 6 & 11 & 7 & \\
\hline 11 & 6 & 5 & 11 & 7 & 5 \\
\hline 11 & 3 & 8 & 12 & 2 & 10 \\
\hline 12 & 3 & 10 & 12 & 4 & 8 \\
\hline 12 & 6 & 7 & 12 & 7 & \\
\hline 12 & 6 & 6 & 12 & 7 & \\
\hline
\end{tabular}

OBS. FREQ. O.-C. TOTAL SEXTIC

\begin{tabular}{|c|c|c|}
\hline 37609.960 & $-\odot .272$ & -0.39 \\
\hline 41516.560 & $-\odot .247$ & -0.37 \\
\hline 41827.870 & -0.206 & -0.39 \\
\hline 58510.300 & 0.112 & -2.62 \\
\hline 58510.300 & ๑. 083 & -2.62 \\
\hline 36198.400 & -0.062 & -0.45 \\
\hline 54222.100 & 0.138 & -1.02 \\
\hline 54245.920 & $-\odot .077$ & -1.02 \\
\hline 38876.260 & 0.058 & -1.20 \\
\hline 50480.830 & -0.257 & -0.63 \\
\hline 58316.850 & 0.023 & -1.01 \\
\hline 58387.420 & $-\odot .030$ & -1.02 \\
\hline 44722.630 & 0.167 & -1.37 \\
\hline 50608.250 & $-\odot .107$ & -1.18 \\
\hline 54959.480 & $-\odot .174$ & -0.94 \\
\hline 29336.500 & 0.104 & 0.16 \\
\hline 28919.160 & $-\odot .081$ & $\odot .26$ \\
\hline 54597.340 & $\odot .098$ & -7.88 \\
\hline 54597.340 & 0.101 & -7.88 \\
\hline 56391.230 & ๑. 019 & -1.17 \\
\hline 59565.860 & $-\odot .235$ & -1.41 \\
\hline 37653.520 & $\odot .048$ & -0.63 \\
\hline 54576.440 & -0.178 & -6.95 \\
\hline 54576.440 & $-\odot .166$ & -6.95 \\
\hline 57754.110 & -0.239 & -3.64 \\
\hline 54384.040 & -0.176 & -3.20 \\
\hline 29472.390 & $-\odot .039$ & 1.11 \\
\hline 54547.750 & $-\odot .298$ & -5.90 \\
\hline 54547.750 & -0.252 & -5.90 \\
\hline 29690.180 & -0.118 & 1.57 \\
\hline 54509.730 & 0.005 & -4.75 \\
\hline 54509.730 & 0.163 & -4.75 \\
\hline 35019.600 & $-\odot .085$ & -8.01 \\
\hline 30080.310 & $-\odot .040$ & 1.93 \\
\hline 54459.470 & -0.187 & -3.49 \\
\hline 54459.470 & ๑. 286 & -3.49 \\
\hline
\end{tabular}




\begin{tabular}{|c|c|c|c|c|c|c|c|c|c|}
\hline 13 & 3 & 11 & 13 & 4 & 9 & 30713.520 & -0.020 & 2.12 & \\
\hline 13 & 4 & 10 & 13 & 5 & 8 & 37347.820 & -0.137 & 2.67 & \\
\hline 13 & 4 & 9 & 13 & 5 & 9 & 36649.360 & -0.130 & 3.22 & \\
\hline 13 & 6 & 7 & 13 & 7 & 7 & 54394.680 & 0.271 & -2.11 & \\
\hline 13 & 3 & 10 & 14 & 2 & 12 & 43615.470 & $\odot . \odot 27$ & -11.16 & \\
\hline 13 & 4 & 9 & 14 & 3 & 11 & 34553.690 & 0.204 & -14.29 & \\
\hline 14 & 3 & 12 & 14 & 4 & 10 & 31672.780 & -0.048 & 2.02 & \\
\hline 14 & 4 & 10 & 14 & 5 & 10 & 36095.460 & $-0.20 \odot$ & 4.67 & \\
\hline 14 & 6 & 9 & 14 & 7 & 7 & 54315.790 & 0.239 & -0.62 & \\
\hline 14 & 6 & 8 & 14 & 7 & 8 & 54312.570 & 0.203 & -0.62 & \\
\hline 14 & 2 & 12 & 15 & 1 & 14 & 39660.420 & -0.148 & -8.26 & \\
\hline 14 & 4 & 10 & 15 & 3 & 12 & 40112.360 & 0.253 & -17.60 & \\
\hline 15 & 3 & 13 & 15 & 4 & 11 & 33048.430 & -0.125 & 1.48 & \\
\hline 15 & 6 & 10 & 15 & 7 & 8 & 54216.890 & -0.064 & 0.99 & \\
\hline 16 & 3 & 14 & 16 & 4 & 12 & 34929.610 & -0.094 & 0.37 & \\
\hline 16 & 4 & 13 & 16 & 5 & 11 & 37515.300 & -0.212 & 5.11 & \\
\hline 16 & 4 & 12 & 16 & 5 & 12 & 34295.850 & -0.281 & 8.61 & \\
\hline 16 & 6 & 11 & 16 & 7 & 9 & 54097.870 & 0.120 & 2.73 & \\
\hline 16 & 6 & 10 & 16 & 7 & 10 & 54081.590 & -0.042 & 2.76 & \\
\hline 16 & 3 & 13 & 17 & 2 & 15 & 52608.990 & 0.215 & -14.75 & \\
\hline 16 & 5 & 12 & 17 & 4 & 14 & 33643.230 & 0.126 & -21.16 & \\
\hline 17 & 4 & 14 & 17 & 5 & 12 & 37848.000 & 0.053 & 5.52 & \\
\hline 17 & 5 & 12 & 17 & 6 & 12 & 44728.540 & -0.089 & 7.58 & \\
\hline 17 & 6 & 12 & 17 & 7 & 10 & 53956.430 & 0.223 & 4.60 & \\
\hline 17 & 6 & 11 & 17 & 7 & 11 & 53922.800 & -0.050 & 4.67 & \\
\hline 17 & 3 & 14 & 18 & 2 & 16 & 54464.500 & 0.278 & -15.65 & \\
\hline 17 & 4 & 13 & 18 & 3 & 15 & 55769.590 & $\odot .301$ & -27.03 & \\
\hline 18 & 3 & 16 & 18 & 4 & 14 & 40484.700 & -0.176 & -3.93 & $\odot .01$ \\
\hline 18 & 6 & 12 & 18 & 7 & 12 & 53725.680 & 0.119 & 6.75 & \\
\hline 19 & 4 & 16 & 19 & 5 & 14 & 39336.460 & $-\odot .090$ & 4.72 & \\
\hline 19 & 6 & 14 & 19 & 7 & 12 & 53603.850 & -0.027 & 8.72 & \\
\hline 19 & 6 & 13 & 19 & 7 & 13 & 53479.070 & -0.200 & 9.04 & \\
\hline 19 & 3 & 17 & 20 & 2 & 19 & 40569.590 & 0.051 & -26.01 & -0.01 \\
\hline 19 & 5 & 14 & 20 & 4 & 16 & 55134.720 & $\odot .356$ & -43.86 & 0.01 \\
\hline 19 & 6 & 13 & 20 & 5 & 15 & 40858.670 & 0.246 & -39.87 & $\odot .01$ \\
\hline 20 & 4 & 17 & 20 & 5 & 15 & 40669.030 & -0.157 & 3.03 & $\odot .01$ \\
\hline 20 & 5 & 16 & 20 & 6 & 14 & 44999.160 & -0.131 & 10.84 & \\
\hline 20 & 6 & 15 & 20 & 7 & 13 & 53396.370 & 0.048 & 10.95 & \\
\hline 20 & 6 & 14 & 20 & 7 & 14 & 53169.460 & -0.014 & 11.60 & \\
\hline 21 & 5 & 16 & 21 & 6 & 16 & 41326.910 & -0.181 & 20.45 & -0.01 \\
\hline 21 & 6 & 16 & 21 & 7 & 14 & 53174.750 & 0.014 & 13.26 & \\
\hline 21 & 6 & 15 & 21 & 7 & 15 & 52776.320 & 0.103 & 14.51 & \\
\hline 21 & 7 & 15 & 22 & 6 & 17 & 38885.340 & -0.112 & -46.68 & \\
\hline 22 & 5 & 17 & 22 & 6 & 17 & 39778.130 & 0.042 & 25.51 & -0.01 \\
\hline 22 & 6 & 17 & 22 & 7 & 15 & 52949.720 & $\odot . \odot 77$ & 15.59 & \\
\hline 22 & 6 & 16 & 22 & 7 & 16 & 52272.390 & -0.126 & 17.89 & \\
\hline 22 & 7 & 16 & 22 & 8 & 14 & 61830.500 & -0.029 & 13.53 & \\
\hline 22 & 7 & 15 & 22 & 8 & 15 & 61780.980 & 0.051 & 13.74 & \\
\hline 22 & 3 & 19 & 23 & 2 & 21 & 58054.130 & $\odot . \odot \odot 9$ & -26.08 & -0.03 \\
\hline 22 & 5 & 18 & 23 & 4 & 20 & 55056.040 & 0.128 & -46.25 & -0.01 \\
\hline 22 & 7 & 15 & 23 & 6 & 17 & 44369.480 & $\odot .095$ & -57.98 & 0.01 \\
\hline 23 & 6 & 18 & 23 & 7 & 16 & 52737.720 & 0.120 & 17.86 & \\
\hline 23 & 6 & 17 & 23 & 7 & 17 & 51622.950 & -0.056 & 21.92 & -0.01 \\
\hline 23 & 7 & 17 & 23 & 8 & 15 & 61602.850 & $\odot .249$ & 16.59 & \\
\hline 23 & 7 & 16 & 23 & 8 & 16 & 61511.460 & 0.170 & 16.99 & \\
\hline 23 & 5 & 19 & 24 & 4 & 21 & 57871.570 & 0.052 & -50.67 & -0.01 \\
\hline 24 & 4 & 21 & 24 & 5 & 19 & 51875.340 & -0.098 & -16.72 & $\odot .04$ \\
\hline
\end{tabular}




\begin{tabular}{|c|c|c|c|c|c|c|c|c|c|}
\hline 24 & 6 & 19 & 24 & 7 & 17 & 52562.820 & $-\odot .023$ & 19.92 & \\
\hline 24 & 6 & 18 & 24 & 7 & 18 & 50783.690 & 0.195 & 26.82 & -0.01 \\
\hline 24 & 7 & 17 & 24 & 8 & 17 & 61186.950 & 0.113 & 20.58 & \\
\hline 24 & 3 & 21 & 25 & 2 & 23 & 58806.470 & $\odot .079$ & -36.77 & -0.04 \\
\hline 24 & 4 & 21 & 25 & 3 & 23 & $55258 . \odot \odot \odot$ & $\odot .189$ & -48.70 & $-\odot .03$ \\
\hline 25 & 5 & 20 & 25 & 6 & 20 & 33049.590 & -0.285 & 44.60 & $-\odot . \odot 4$ \\
\hline 25 & 6 & 20 & 25 & 7 & 18 & 52458.840 & -0.183 & 21.57 & \\
\hline 24 & 9 & 16 & 25 & 8 & 18 & 33999.930 & -0.308 & -61.23 & 0.01 \\
\hline 25 & 7 & 18 & 25 & 8 & 18 & 60793.150 & 0.344 & 24.58 & -0.01 \\
\hline 26 & 4 & 23 & 26 & 5 & 21 & 61476.320 & $-\odot .04 \odot$ & -33.59 & 0.08 \\
\hline 25 & 7 & 18 & 26 & 6 & 20 & 60252.140 & 0.182 & -94.38 & 0.03 \\
\hline 26 & 5 & 21 & 26 & 6 & 21 & 30204.020 & -0.169 & 51.38 & -0.05 \\
\hline 26 & 6 & 21 & 26 & 7 & 19 & 52470.840 & -0.056 & 22.51 & \\
\hline 26 & 7 & 19 & 26 & 8 & 19 & 60309.390 & 0.283 & 29.16 & -0.01 \\
\hline 26 & 4 & 23 & 27 & 3 & 25 & 58098.120 & 0.145 & -60.27 & -0.05 \\
\hline 27 & 5 & 23 & 27 & 6 & 21 & 52152.460 & -0.144 & -8.40 & 0.05 \\
\hline 27 & 5 & 23 & 27 & 6 & 21 & 52152.460 & -0.144 & -8.40 & 0.05 \\
\hline 27 & 5 & 22 & 27 & 6 & 22 & 27157.720 & -0.093 & 57.77 & -0.06 \\
\hline 26 & 8 & 18 & 27 & 7 & 20 & 53054.340 & 0.118 & -93.86 & 0.02 \\
\hline 27 & 6 & 22 & 27 & 7 & 20 & 52655.500 & -0.273 & 22.36 & 0.01 \\
\hline 27 & 7 & 21 & 27 & 8 & 19 & $6 \odot 49 \odot .52 \odot$ & $\odot .06 \odot$ & 29.87 & -0.01 \\
\hline 27 & 3 & 24 & 28 & 2 & 26 & 60747.340 & $-\odot .249$ & -59.56 & $-\odot .08$ \\
\hline 28 & 5 & 24 & 28 & 6 & 22 & 55236.820 & -0.222 & -19.08 & $\odot .08$ \\
\hline 27 & 8 & 19 & 28 & 7 & 21 & 58049.210 & 0.047 & -108.17 & 0.03 \\
\hline 28 & 6 & 23 & 28 & 7 & 21 & 53084.550 & 0.149 & 20.64 & 0.01 \\
\hline 27 & 8 & 20 & 28 & 7 & 22 & 56966.340 & 0.320 & -101.58 & 0.02 \\
\hline 28 & 7 & 22 & 28 & 8 & 20 & $6 \odot 2 \odot 7.50 \odot$ & 0.066 & 33.06 & -0.01 \\
\hline 28 & 7 & 21 & 28 & 8 & 21 & 58956.970 & $\odot .220$ & $4 \odot .87$ & $-\odot .02$ \\
\hline 28 & 3 & 25 & 29 & 2 & 27 & 61609.340 & $-\odot .089$ & -68.48 & $-\odot .09$ \\
\hline 29 & 5 & 25 & 29 & 6 & 23 & 59046.440 & 0.225 & -32.02 & 0.11 \\
\hline 29 & 6 & 24 & 29 & 7 & 22 & 53840.890 & 0.217 & 16.74 & 0.02 \\
\hline 28 & 8 & 21 & 29 & 7 & 23 & 61567.170 & -0.047 & -113.81 & 0.02 \\
\hline 29 & 7 & 23 & 29 & 8 & 21 & 59959.770 & -0.234 & 35.84 & -0.01 \\
\hline 29 & 4 & 26 & 30 & 3 & 28 & 61908.910 & 0.074 & -82.68 & -0.10 \\
\hline 30 & 6 & 24 & 30 & 7 & 24 & 39167.030 & $-\odot .007$ & 81.98 & -0.10 \\
\hline 30 & 7 & 24 & 30 & 8 & 22 & 59781.070 & -0.060 & 37.91 & \\
\hline 29 & 9 & 21 & 30 & 8 & 23 & 56665.850 & 0.198 & -122.98 & $\odot .02$ \\
\hline 30 & 7 & 23 & 30 & 8 & 23 & 56813.090 & -0.123 & 58.17 & -0.04 \\
\hline 31 & 6 & 26 & 31 & 7 & 24 & 56720.700 & 0.080 & -0.10 & 0.07 \\
\hline 31 & 7 & 25 & 31 & 8 & 23 & 59714.460 & -0.110 & 38.82 & \\
\hline 30 & 9 & 22 & 31 & 8 & 24 & 61306.900 & 0.284 & -137.93 & 0.03 \\
\hline 32 & 6 & 27 & 32 & 7 & 25 & 59041.400 & 0.119 & -14.10 & 0.11 \\
\hline 32 & 6 & 26 & 32 & 7 & 26 & 32584.580 & ๑.011 & 104.60 & -0.15 \\
\hline 31 & 10 & 21 & 32 & 9 & 23 & 56346.680 & 0.114 & -146.15 & 0.03 \\
\hline 33 & 6 & 27 & 33 & 7 & 27 & 29010.180 & -0.018 & 114.15 & -0.18 \\
\hline 32 & 10 & 23 & 33 & 9 & 25 & 60882.050 & 0.175 & -162.70 & 0.04 \\
\hline 34 & 7 & 28 & 34 & 8 & 26 & 60816.700 & ๑. 087 & 28.15 & 0.05 \\
\hline 33 & 11 & 22 & 34 & 10 & 24 & 55932.340 & 0.040 & -169.59 & 0.04 \\
\hline 33 & 11 & 23 & 34 & 10 & 25 & 55916.870 & 0.198 & -169.37 & 0.04 \\
\hline 34 & 11 & 24 & 35 & 10 & 26 & 60471.210 & -0.132 & -188.95 & 0.05 \\
\hline 35 & 12 & 24 & 36 & 11 & 26 & 55604.720 & 0.228 & -195.25 & 0.05 \\
\hline 36 & 12 & 24 & 37 & 11 & 26 & 60119.290 & -0.137 & -217.24 & 0.06 \\
\hline 36 & 12 & 25 & 37 & 11 & 27 & 60113.290 & -0.208 & -217.14 & 0.06 \\
\hline 39 & 7 & 32 & 39 & 8 & 32 & 30270.320 & 0.033 & 201.34 & -0.47 \\
\hline 38 & 14 & 24 & 39 & 13 & 26 & 50672.390 & -0.170 & -227.47 & 0.06 \\
\hline 38 & 14 & 25 & 39 & 13 & 27 & 50672.390 & -0.103 & -227.47 & 0.06 \\
\hline 40 & 7 & 33 & 40 & 8 & 33 & 26217.800 & -0.033 & 209.34 & -0.53 \\
\hline
\end{tabular}




\begin{tabular}{|c|c|c|c|c|c|c|c|c|c|}
\hline 40 & 15 & 25 & 41 & 14 & 27 & 50475.060 & -0.332 & -256.80 & $\odot .07$ \\
\hline 40 & 15 & 26 & 41 & 14 & 28 & 50475.060 & -0.320 & -256.80 & 0.07 \\
\hline 42 & 8 & 34 & 42 & 9 & 34 & 44195.300 & 0.060 & 269.18 & -0.69 \\
\hline 41 & 15 & 26 & 42 & 14 & 28 & 54871.520 & $\odot .074$ & -285.05 & 0.08 \\
\hline 41 & 15 & 27 & 42 & 14 & 29 & 54871.520 & $\odot .098$ & -285.04 & $\odot . \odot 8$ \\
\hline 43 & 8 & 35 & 43 & 9 & 35 & 39920.260 & -0.011 & 293.55 & -0.82 \\
\hline 42 & 15 & 27 & 43 & 14 & 29 & 59286.570 & -0.305 & -314.65 & 0.10 \\
\hline 42 & 15 & 28 & 43 & 14 & 30 & 59286.570 & -0.259 & -314.65 & 0.10 \\
\hline 42 & 16 & 26 & 43 & 15 & 28 & 50290.910 & -0.229 & -288.06 & 0.08 \\
\hline 42 & 16 & 27 & 43 & 15 & 29 & 50290.910 & -0.227 & -288.06 & $\odot . \odot 8$ \\
\hline 44 & 8 & 36 & 44 & 9 & 36 & 35489.670 & -0.048 & 313.12 & -0.94 \\
\hline 43 & 16 & 27 & 44 & 15 & 29 & 54667.900 & -0.104 & -319.12 & 0.10 \\
\hline 43 & 16 & 28 & 44 & 15 & 30 & 54667.900 & $-\odot . \odot 99$ & -319.12 & 0.10 \\
\hline 45 & 8 & 37 & 45 & 9 & 37 & 31015.980 & 0.060 & 326.41 & -1.05 \\
\hline 45 & 9 & 36 & 45 & 10 & 36 & 58463.460 & -0.281 & 311.91 & $-\odot .85$ \\
\hline 46 & 8 & 38 & 46 & 9 & 38 & 26614.890 & 0.105 & 332.14 & -1.13 \\
\hline 45 & 17 & 28 & 46 & 16 & 30 & 54476.540 & -0.088 & -355.28 & 0.12 \\
\hline 45 & 17 & 29 & 46 & 16 & 31 & 54476.540 & -0.088 & -355.28 & 0.12 \\
\hline 46 & 17 & 29 & 47 & 16 & 31 & 58851.980 & 0.027 & -390.75 & 0.14 \\
\hline 46 & 17 & 30 & 47 & 16 & 32 & 58851.980 & $\odot .028$ & -390.75 & 0.14 \\
\hline 47 & 18 & 29 & 48 & 17 & 31 & 54294.180 & -0.125 & -393.55 & 0.14 \\
\hline 47 & 18 & 30 & 48 & 17 & 32 & 54294.180 & -0.125 & -393.55 & 0.14 \\
\hline 49 & 9 & 40 & 49 & 10 & 40 & 41022.700 & 0.016 & 463.10 & -1.73 \\
\hline 48 & 18 & 30 & 49 & 17 & 32 & 58653.300 & 0.011 & -432.17 & 0.16 \\
\hline 48 & 18 & 31 & 49 & 17 & 33 & 58653.300 & $\odot .011$ & -432.17 & $\odot .16$ \\
\hline 50 & 9 & 41 & 50 & 10 & 41 & 36156.220 & 0.180 & 484.64 & -1.93 \\
\hline 49 & 19 & 30 & 50 & 18 & 32 & 54118.550 & -0.260 & -433.95 & 0.16 \\
\hline 49 & 19 & 31 & 50 & 18 & 33 & 54118.550 & -0.260 & -433.95 & $\odot .16$ \\
\hline 51 & 9 & 42 & 51 & 10 & 42 & 31318.970 & -0.017 & 495.56 & -2.09 \\
\hline 52 & 10 & 42 & 52 & 11 & 42 & 56748.180 & 0.043 & 557.42 & -2.23 \\
\hline 52 & 20 & 32 & 53 & 19 & 34 & 58279.770 & $\odot .159$ & -521.81 & 0.22 \\
\hline 52 & 20 & 33 & 53 & 19 & 35 & 58279.770 & 0.159 & -521.81 & 0.22 \\
\hline 53 & 21 & 32 & 54 & 20 & 34 & 53782.020 & 0.041 & -521.18 & 0.23 \\
\hline 53 & 21 & 33 & 54 & 20 & 35 & 53782.020 & 0.041 & -521.18 & 0.23 \\
\hline 55 & 10 & 45 & 55 & 11 & 45 & 41634.320 & -0.106 & 690.95 & -3.34 \\
\hline 54 & 21 & 33 & 55 & 20 & 35 & 58101.130 & 0.153 & -570.07 & 0.26 \\
\hline 54 & 21 & 34 & 55 & 20 & 36 & 58101.130 & 0.153 & -570.07 & $\odot .26$ \\
\hline 56 & 10 & 46 & 56 & 11 & 46 & 36386.410 & 0.214 & 710.48 & -3.62 \\
\hline 55 & 22 & 33 & 56 & 21 & 35 & 53618.370 & 0.033 & -568.06 & 0.27 \\
\hline 55 & 22 & 34 & 56 & 21 & 36 & 53618.370 & 0.033 & -568.06 & 0.27 \\
\hline 57 & 10 & 47 & 57 & 11 & 47 & 31247.110 & -0.180 & 713.58 & -3.82 \\
\hline 58 & 10 & 48 & 58 & 11 & 48 & 26344.220 & -0.100 & 698.97 & -3.92 \\
\hline 59 & 11 & 48 & 59 & 12 & 48 & 52995.490 & $\odot .009$ & 900.20 & -4.92 \\
\hline 62 & 11 & 51 & 62 & 12 & 51 & 36243.200 & -0.119 & 995.93 & -6.33 \\
\hline 61 & 24 & 37 & 62 & 23 & 39 & 61879.050 & 0.174 & -791.14 & 0.45 \\
\hline 61 & 24 & 38 & 62 & 23 & 40 & 61879.050 & 0.174 & -791.14 & 0.45 \\
\hline 63 & 11 & 52 & 63 & 12 & 52 & 30865.040 & $\odot .024$ & 983.49 & -6.53 \\
\hline 64 & 11 & 53 & 64 & 12 & 53 & 25807.520 & 0.228 & 946.83 & -6.55 \\
\hline 64 & 12 & 52 & 64 & 13 & 52 & 59412.890 & -0.112 & 1196.56 & -7.71 \\
\hline 65 & 12 & 53 & 65 & 13 & 53 & 53579.770 & 0.039 & 1272.70 & -8.64 \\
\hline
\end{tabular}

STOPPED ON DIVERGENCY

RMS $=0.1638 \quad$ GRAMDET.$=0.31 \mathrm{E}-12$

ROTATIONAL CONSTANTS (MHZ): 6271.536383 2244.438353

1886.235519 

$+-$
$\odot .002964$
0.001345
0.001283

\begin{tabular}{|c|c|c|c|c|}
\hline QUARTIC & DISTORTION & CONSTANTS: & & \\
\hline & $\odot .90064007$ & -4.00181631 & 10.53236558 & 0.04164010 \\
\hline+- & ๑. $00 \odot 85264$ & ๑.0०392672 & ๑. 01492581 & 0.00021545 \\
\hline SEXTIC & DISTORTION & CONSTANTS: & & \\
\hline & $\odot .0 \odot \odot \odot \odot \odot \odot \odot$ & 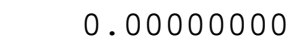 & 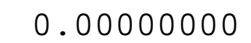 & $\odot .00000000$ \\
\hline+- & $\odot . \odot \odot \odot \odot \odot \odot \odot \odot$ & $\odot . \odot \odot \odot \odot \odot \odot \odot \odot$ & $\odot . \odot \odot \odot \odot \odot \odot \odot \odot$ & $\odot . ๑ \odot \odot \odot \odot \odot \odot \odot$ \\
\hline & $\odot .0 \odot \odot 21703$ & $\odot . \odot \odot \odot \odot \odot \odot \odot \odot ~$ & $\odot . ๑ \odot \odot \odot \odot \odot \odot \odot ~$ & \\
\hline+- & ๑. .०००19๑6 & ๑. ๑૦९९९९७९ & 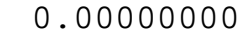 & \\
\hline
\end{tabular}

SIGNIFICANT DIGITS AND CORRELATION MATRIX:

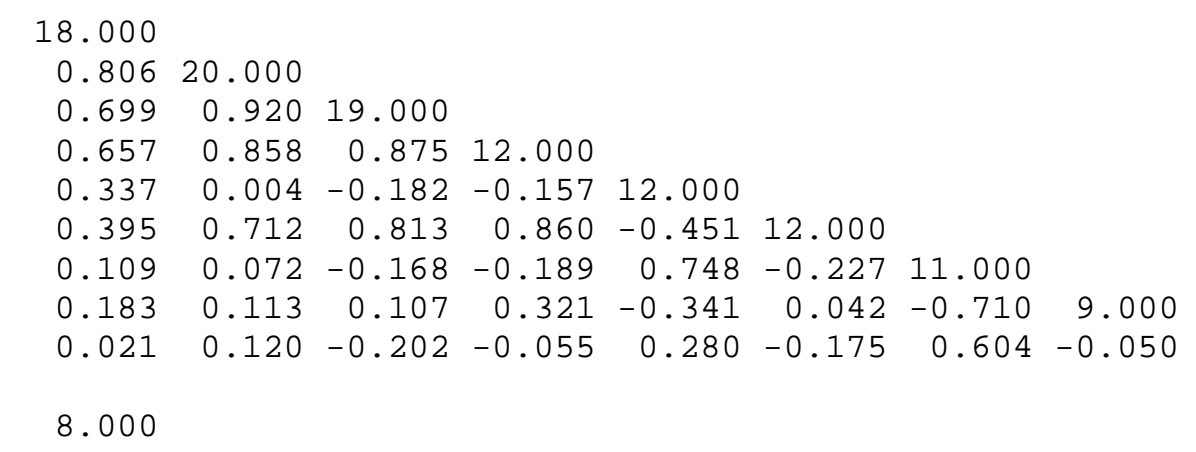

\section{DERIVED CONSTANTS:}

KAPPA $=-0.836635$

MOMENTS OF INERTIA AND DEFECT $\left(U^{*} A * * 2\right)$ : $80.58297347225 .16949477267 .92998272-37.82248552$

$\begin{array}{lllll}+- & 0.00003808 & 0.00013497 & 0.00018227 & 0.00007879\end{array}$

STANDARD DISTORTION CONSTANTS (KHZ):

$\begin{array}{rrr}-29.724757 & -3.935681 & -3.269439 \\ 2.359608 & -3.602560 & 6.442537\end{array}$


TABLE 4S: Microwave Spectrum of the First Excited State of the Lowest Bending Vibration of Conformer I of Cyclopentadienylphosphine

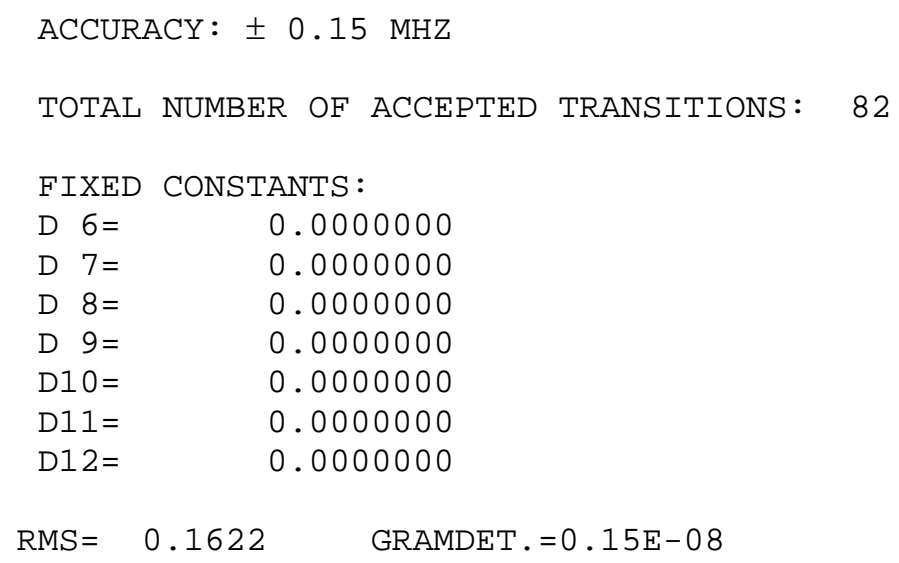

TRANSITION

\begin{tabular}{|c|c|c|c|c|c|}
\hline & & & \multicolumn{3}{|c|}{$J^{\prime}{ }_{k-1}^{\prime}{ }^{\prime}{ }^{\prime}{ }^{\prime}$} \\
\hline 4 & 0 & 4 & 5 & 1 & 4 \\
\hline 4 & 1 & 3 & 5 & 2 & \\
\hline 5 & $\odot$ & 5 & 6 & & \\
\hline 5 & 1 & 4 & & 2 & \\
\hline 5 & 3 & 2 & 6 & 4 & \\
\hline 6 & 1 & 5 & 7 & 2 & \\
\hline 6 & 3 & 4 & 7 & 4 & \\
\hline 7 & 2 & 5 & 8 & 3 & \\
\hline 9 & 3 & 7 & 9 & 4 & \\
\hline 9 & 3 & 6 & 9 & 4 & \\
\hline 9 & 2 & 7 & 10 & 3 & \\
\hline 10 & 3 & 8 & 10 & 4 & \\
\hline 10 & 1 & 9 & 11 & 2 & \\
\hline 11 & 3 & 9 & 11 & 4 & \\
\hline 11 & 6 & 5 & 11 & 7 & \\
\hline 11 & 6 & 5 & 11 & 7 & \\
\hline 12 & 6 & 7 & 12 & 7 & \\
\hline 12 & 6 & 6 & 12 & 7 & \\
\hline 13 & 4 & 9 & 13 & 5 & \\
\hline 14 & 3 & 12 & 14 & 4 & 10 \\
\hline 14 & 4 & 10 & 14 & 5 & \\
\hline 15 & 6 & 10 & 15 & 7 & \\
\hline 15 & 6 & 9 & 15 & 7 & \\
\hline 16 & 6 & 11 & 16 & 7 & \\
\hline 16 & 6 & 10 & 16 & 7 & 10 \\
\hline 17 & 4 & 14 & 17 & 5 & \\
\hline 17 & 6 & 12 & 17 & 7 & 10 \\
\hline 17 & 6 & 11 & 17 & 7 & \\
\hline 18 & 5 & 13 & 18 & 6 & \\
\hline 18 & 6 & 12 & 18 & 7 & \\
\hline 18 & 3 & 15 & 19 & 2 & \\
\hline 18 & 4 & 14 & 19 & 3 & \\
\hline 19 & 3 & 17 & 19 & 4 & \\
\hline 19 & 4 & 16 & 19 & 5 & \\
\hline 19 & 6 & 14 & 19 & 7 & \\
\hline
\end{tabular}

OBS. FREQ. O. - C. TOTAL

DISTORTION CONTRIBUTION

\begin{tabular}{|c|c|c|}
\hline 27716.450 & -0.148 & -0.38 \\
\hline 32252.940 & 0.032 & -0.23 \\
\hline 33144.570 & 0.226 & -0.70 \\
\hline 36210.670 & 0.241 & -0.44 \\
\hline 54227.420 & $\odot .0 \odot 4$ & -0.94 \\
\hline 40352.650 & -0.149 & -0.79 \\
\hline 58395.410 & -0.047 & -0.96 \\
\hline 52845.770 & -0.016 & $-\odot .78$ \\
\hline 29358.010 & -0.037 & $\odot .61$ \\
\hline 28541.030 & -0.167 & 0.83 \\
\hline 59973.570 & -0.010 & -1.76 \\
\hline 29460.320 & $-0.24 \odot$ & 1.06 \\
\hline 59740.840 & -0.196 & -4.42 \\
\hline 29677.380 & 0.110 & 1.48 \\
\hline 54490.370 & 0.149 & -4.21 \\
\hline 54490.370 & 0.149 & -4.21 \\
\hline 54440.160 & -0.198 & -3.04 \\
\hline 54440.160 & 0.271 & -3.04 \\
\hline 36639.230 & -0.245 & 3.05 \\
\hline 31651.010 & -0.072 & 1.88 \\
\hline 36087.680 & 0.020 & 4.39 \\
\hline $54198 . \odot \odot \odot$ & $-\odot . \odot \odot 9$ & 1.10 \\
\hline 54190.840 & 0.161 & 1.12 \\
\hline 54079.110 & 0.105 & 2.71 \\
\hline 54062.790 & -0.216 & 2.74 \\
\hline 37829.270 & 0.200 & 5.16 \\
\hline 53937.370 & -0.332 & 4.44 \\
\hline 53904.750 & 0.159 & 4.51 \\
\hline 44189.010 & -0.111 & 9.31 \\
\hline 53707.590 & -0.221 & 6.44 \\
\hline 55900.600 & $-\odot .086$ & -16.15 \\
\hline 60274.890 & 0.219 & -28.20 \\
\hline 44162.620 & 0.135 & -6.82 \\
\hline 39309.230 & $\odot .308$ & 4.39 \\
\hline 53585.950 & $\odot .019$ & 8.26 \\
\hline
\end{tabular}




\begin{tabular}{|c|c|c|c|c|c|c|c|}
\hline 19 & 613 & 19 & 7 & 13 & 53462.440 & 0.211 & 8.56 \\
\hline 19 & $\begin{array}{ll}5 & 14\end{array}$ & 20 & $4:$ & 16 & 55177.770 & -0.013 & -41.87 \\
\hline 20 & 417 & 20 & 5 & 15 & 40634.840 & 0.112 & 2.79 \\
\hline 20 & 416 & 20 & 5 & 16 & 27114.790 & 0.016 & 19.83 \\
\hline 20 & 615 & $2 \odot$ & 7 & 13 & 53378.700 & $\odot .06 \odot$ & 10.33 \\
\hline 20 & 614 & 20 & 7 & 14 & 53153.700 & 0.279 & 10.93 \\
\hline 21 & 616 & 21 & 7 & 14 & 53157.120 & -0.118 & 12.47 \\
\hline 21 & $\begin{array}{ll}7 & 14\end{array}$ & 21 & 8 & 14 & 61985.730 & $-\odot .065$ & 10.23 \\
\hline 22 & 17 & 22 & 7 & 15 & 52932.220 & 0.055 & 14.64 \\
\hline 23 & 18 & 23 & 7 & 16 & 52719.850 & $-\odot . \odot \odot 6$ & 16.74 \\
\hline 23 & $\begin{array}{ll}6 & 17\end{array}$ & 23 & 7 & 17 & 51612.770 & -0.137 & 20.53 \\
\hline 23 & 716 & 23 & 8 & 16 & 61491.480 & -0.278 & 16.03 \\
\hline 23 & 320 & 24 & 2 & 22 & 58527.210 & $\odot .064$ & -29.89 \\
\hline 24 & 421 & 24 & 5 & 19 & 51796.340 & -0.010 & -15.87 \\
\hline 24 & 619 & 24 & 7 & 17 & 52544.540 & 0.152 & 18.65 \\
\hline 24 & 618 & 24 & 7 & 18 & 50777.080 & 0.108 & 25.09 \\
\hline 24 & 17 & 24 & 8 & 17 & 61168.270 & 0.016 & 19.36 \\
\hline 24 & 20 & 25 & 4 & 22 & 60560.590 & $-\odot .092$ & -53.00 \\
\hline 25 & 4 & 25 & 5 & 20 & 56262.090 & -0.294 & -23.50 \\
\hline 26 & 521 & 26 & 6 & 21 & 30236.710 & -0.117 & 48.12 \\
\hline 26 & 21 & 26 & 7 & 19 & 52448.670 & -0.123 & 21.04 \\
\hline 26 & $7 \quad 19$ & 26 & 8 & 19 & 60293.410 & -0.142 & 27.32 \\
\hline 27 & 523 & 27 & 6 & 21 & 52088.800 & -0.036 & -8.03 \\
\hline 27 & 522 & 27 & 6 & 22 & 27195.770 & 0.101 & 54.14 \\
\hline 27 & 6 & 27 & 7 & 20 & $52630.00 \odot$ & -0.170 & 20.88 \\
\hline 27 & 720 & 27 & 8 & 20 & $59695.60 \odot$ & $-\odot .022$ & 32.29 \\
\hline 28 & 524 & 28 & 6 & 22 & 55159.220 & 0.288 & -18.10 \\
\hline 28 & 722 & 28 & 8 & 20 & 60187.890 & $\odot .093$ & 30.94 \\
\hline 29 & 624 & 29 & 7 & 22 & 53803.240 & 0.232 & 15.58 \\
\hline 29 & 7 & 29 & 8 & 21 & 59939.390 & -0.163 & 33.53 \\
\hline 29 & 722 & 29 & 8 & 22 & 58003.040 & -0.109 & 45.47 \\
\hline 30 & 724 & 30 & 8 & 22 & 59759.230 & 0.099 & 35.44 \\
\hline 31 & 626 & 31 & 7 & 24 & 56662.000 & $\odot .0 \odot 3$ & -0.26 \\
\hline 31 & 625 & 31 & 7 & 25 & 36042.210 & 0.133 & 87.75 \\
\hline 31 & 24 & 31 & 8 & 24 & 55320.130 & 0.067 & 65.25 \\
\hline 32 & 626 & 32 & 7 & 26 & 32634.430 & -0.117 & 98.08 \\
\hline 32 & 725 & 32 & 8 & 25 & 53470.820 & 0.086 & 78.31 \\
\hline 33 & 6 & 33 & 7 & 26 & 61973.170 & -0.167 & -30.37 \\
\hline 33 & 627 & 33 & 7 & 27 & 29065.520 & 0.131 & 107.11 \\
\hline 35 & 29 & 35 & 8 & 27 & 61840.700 & $-\odot . \odot \odot 2$ & 16.06 \\
\hline 37 & 730 & 37 & 8 & 30 & 38328.660 & 0.029 & 162.77 \\
\hline 37 & 829 & 37 & 9 & 29 & $6 \odot 357.920$ & 0.049 & 123.63 \\
\hline 40 & 733 & 40 & 8 & 33 & 26293.140 & -0.324 & 196.87 \\
\hline 42 & 834 & 42 & 9 & 34 & 44268.940 & $-\odot .028$ & 252.61 \\
\hline 43 & 35 & 43 & 9 & 35 & $40 \odot \odot 2.5 \odot \odot$ & 0.247 & 275.73 \\
\hline 45 & $8 \quad 37$ & 45 & 9 & 37 & 31107.110 & $-\odot .087$ & 307.15 \\
\hline 16 & 838 & 46 & 9 & 38 & 26706.560 & -0.004 & 312.84 \\
\hline
\end{tabular}

RMS $=0.1622 \quad$ GRAMDET.$=0.15 \mathrm{E}-08$

ROTATIONAL CONSTANTS (MHZ):

$\begin{array}{rrrr}6271.329814 & 2245.513776 & 1887.878816 \\ +- & 0.006247 & 0.003728 & 0.003937\end{array}$

QUARTIC DISTORTION CONSTANTS: 


$\begin{array}{rrrrrr} & 0.86575546 & -3.69525453 & 9.56622967 & 0.04051410 & 0.39625855 \\ +- & 0.00430166 & 0.01259425 & 0.09419243 & 0.00061022 & 0.03579983\end{array}$

SIGNIFICANT DIGITS AND CORRELATION MATRIX:
18.000
0.32619 .000
$0.237 \quad 0.982 \quad 19.000$
$\begin{array}{llll}0.171 & 0.898 & 0.917 & 9.000\end{array}$
$\begin{array}{lllll}-0.094 & 0.183 & 0.214 & 0.172 & 11.000\end{array}$
$\begin{array}{llllll}0.636 & -0.271 & -0.329 & -0.331 & -0.656 & 9.000\end{array}$
$\begin{array}{lllllll}-0.013 & 0.167 & 0.194 & 0.130 & 0.941 & -0.482 & 10.000\end{array}$
$\begin{array}{llllllll}0.097 & -0.193 & -0.262 & -0.187 & -0.870 & 0.487 & -0.957 & 8.00 \odot\end{array}$
DERIVED CONSTANTS:
KAPPA $=-0.836825$
MOMENTS OF INERTIA AND DEFECT $\left(U^{*} A * * 2\right)$ :
$80.58562777225 .06165645 \quad 267.69676406-37.95052015$
+- $\quad 0.00008027 \quad 0.00037365 \quad 0.00055824 \quad 0.00021524$
STANDARD DISTORTION CONSTANTS (KHZ):

$\begin{array}{rrr}-26.946922 & -3.787135 & -3.138909 \\ 2.180397 & -3.463022 & 5.674578\end{array}$


TABLE 5S: MP2/aug-cc-pVTZ and B3LYP/6-311++G(d,p) Structures ${ }^{a}$ of Conformers I and II of Cyclopentadienylphosphine

\begin{tabular}{|c|c|c|c|c|}
\hline \multirow{2}{*}{$\begin{array}{l}\text { conformer } \\
\text { method }\end{array}$} & \multicolumn{2}{|c|}{ I } & \multicolumn{2}{|c|}{ II } \\
\hline & MP2 & B3LYP & MP2 & B3LYP \\
\hline \multicolumn{5}{|c|}{ bond length (pm) } \\
\hline C1-C2 & 136.0 & 135.0 & 135.8 & 135.0 \\
\hline C2-H5 & 108.0 & 108.2 & 108.0 & 108.1 \\
\hline C1-C9 & 148.8 & 150.0 & 148.7 & 149.9 \\
\hline C2-C3 & 145.1 & 146.3 & 145.6 & 146.5 \\
\hline C2-H6 & 108.0 & 108.2 & 108.0 & 108.2 \\
\hline C3-C4 & 136.0 & 135.0 & 135.7 & 134.9 \\
\hline C3-H7 & 108.0 & 108.2 & 108.0 & 108.2 \\
\hline C4-H8 & 108.0 & 108.2 & 108.0 & 108.1 \\
\hline C4-C9 & 148.8 & 150.0 & 149.3 & 150.4 \\
\hline C9-H10 & 108.9 & 109.4 & 109.4 & 109.8 \\
\hline C9-P11 & 188.6 & 190.5 & 188.3 & 190.1 \\
\hline P11-H12 & 141.8 & 142.7 & 141.7 & 142.6 \\
\hline P11-H13 & 141.8 & 142.7 & 141.5 & 142.3 \\
\hline \multicolumn{5}{|c|}{ angles(deg) } \\
\hline C2-C1-H5 & 127.0 & 126.9 & 127.1 & 127.1 \\
\hline
\end{tabular}


(Table 1 continued; second page)

\begin{tabular}{|c|c|c|c|c|}
\hline C2-C1-C9 & 108.9 & 109.2 & 109.1 & 109.2 \\
\hline H5-C1-C9 & 124.1 & 123.9 & 123.8 & 123.6 \\
\hline C1-C2-C3 & 109.2 & 109.2 & 109.0 & 109.1 \\
\hline C1-C2-H6 & 125.8 & 126.1 & 125.9 & 126.1 \\
\hline C3-C2-H6 & 125.1 & 124.7 & 125.0 & 124.7 \\
\hline C2-C3-C4 & 109.2 & 109.2 & 109.2 & 109.3 \\
\hline C2-C3-H7 & 125.0 & 124.7 & 125.0 & 124.7 \\
\hline C4-C3-H7 & 125.8 & 126.1 & 125.7 & 126.0 \\
\hline C3-C4-H8 & 127.0 & 126.9 & 127.1 & 127.1 \\
\hline C3-C4-C9 & 108.9 & 109.2 & 108.8 & 109.0 \\
\hline H8-C4-C9 & 124.1 & 123.9 & 124.1 & 123.8 \\
\hline C1-C9-C4 & 103.9 & 103.2 & 103.8 & 103.2 \\
\hline C1-C9-H10 & 115.5 & 113.1 & 113.9 & 112.2 \\
\hline C1-C9-P11 & 105.3 & 109.3 & 112.7 & 115.1 \\
\hline C4-C9-H10 & 115.6 & 113.1 & 113.4 & 111.8 \\
\hline C4-C9-P11 & 105.3 & 109.4 & 107.5 & 110.3 \\
\hline H10-С9-P11 & 110.2 & 108.9 & 105.6 & 104.4 \\
\hline
\end{tabular}

(Table 1 continues next page) 
(Table 1 continued; third page)

$\begin{array}{lllll}\text { C9-P11-H12 } & 96.9 & 96.4 & 97.0 & 96.8 \\ \text { C9-P11-H13 } & 96.9 & 96.4 & 95.2 & 96.5 \\ \text { H12-P11-H13 } & 92.8 & 92.7 & 93.9 & 93.8\end{array}$

\begin{tabular}{lrrrr} 
& \multicolumn{4}{c}{${\text { dihedral } \text { angles }^{b} \text { (deg) }}$} \\
H5-C1-C2-C3 & -179.5 & 179.8 & -179.0 & -179.8 \\
C9-C1-C2-C3 & -2.1 & -1.2 & -1.4 & -0.8 \\
C2-C1-C9-C4 & 3.2 & 1.8 & 2.3 & 1.2 \\
C2-C1-C9-H10 & 130.9 & 124.4 & 126.0 & 121.7 \\
C2-C1-C9-P11 & -107.2 & -114.5 & -113.7 & -119.1 \\
C1-C2-C3-C4 & 0.0 & 0.0 & -0.1 & 0.0 \\
C1-C2-C3-H7 & 179.0 & 180.0 & 179.5 & 180.0 \\
H6-C2-C3-C4 & -178.9 & 180.0 & -178.9 & -179.6 \\
C2-C3-C4-H8 & 179.3 & -179.8 & 179.8 & -179.7 \\
C2-C3-C4-C9 & 2.1 & 1.1 & 1.6 & 0.8 \\
C3-C4-C9-C1 & -3.2 & -1.8 & -2.3 & -1.2 \\
C3-C4-C9-P11 & 107.2 & 114.5 & 117.2 & 122.3 \\
C1-C9-P11-H12 & -172.2 & -170.6 & -49.9 & -50.3 \\
C1-C9-P11-H13 & -78.5 & -77.2 & 44.7 & 44.3
\end{tabular}

${ }^{a}$ Atom numbering is given in Fig. $1 .^{b}$ The dihedral angle is $0^{\circ}$ in the synperiplanar conformation. 